\title{
Phenomenological Renormalization Group Methods
}

\author{
J. A. Plascak, \\ Departamento de Fúsica, Instituto de Ciências Exatas, \\ Universidade Federal de Minas Gerais, C.P. 702, \\ 30161-123 Belo Horizonte, Brazil \\ W. Figueiredo, and B. C. S. Grandi \\ Departamento de Fisica, \\ Universidade Federal de Santa Catarina \\ 88040-900 Florianópolis, Brazil \\ Received 26 May, 1999
}

\begin{abstract}
Some renormalization group approaches have been proposed during the last few years which are close in spirit to the Nightingale phenomenological procedure. In essence, by exploiting the finite size scaling hypothesis, the approximate critical behavior of model on infinite lattice is obtained through the exact computation of some thermal quantities of the model on finite clusters. In this work some of these methods are reviewed, namely the mean field renormalization group, the effective field renormalization group and the finite size scaling renormalization group procedures. Although special emphasis is given to the mean field renormalization group (since it has been, up to now, much more used to study a wide variety of different systems) a discussion of their potentialities and interrelations to other methods is also presented.
\end{abstract}

\section{Introduction}

The renormalization group formalism introduced by Wilson in the early 70's $[1,2]$ is by now one of the basic strategies to solve fundamental problems in statistical mechanics. It is also a very useful tool to tackle problems in several fields of theoretical physics such as the study of nonlinear dynamics and transitions to chaos [3], disorder surface growth [4], earthquakes [5], among others. The conceptual foundation of the method, first laid by Kadanoff [6] to qualitatively predict scaling behavior at a second-order phase transition, is to reduce, in a step-by-step way, the degrees of freedom of the system leaving unchanged the underlying physics of the problem. This reduction, carried out repeatedly through a renormalization recursion relation, leads the original system with a large correlation length (the range at which physical microscopic operators are correlated) to one with correlation length of unity order, where well-known methods as perturbation theory can, at least in principle, be used to treat the problem. Depending on the mathematical technique, such thinning of the degrees of freedom can be implemented directly in the reciprocal (momentum) space or in the real (position) space. The former approach makes use of mathematical tools from quantum field theory with the crystalline system being replaced by its continuous limit. As a result, the so-called $\epsilon$-expansion proposed by Wilson and Fisher [7] (and further developed by using techniques of renormalized perturbation theory $[8,9]$ ) provides analytical and quite well controlled asymptotically exact results for critical exponents (despite being unable to predict values of critical points, critical lines and phase diagrams). On the other hand, the more intuitive real space version of the renormalization group works directly in the position space. It was introduced by Niemeijer and van Leeuwen [10] and several different techniques have been proposed and applied to a great variety of statistical models [11]. The real space renormalization group (RSRG) has since become an important apparatus in studying critical phenomena.

The main feature of the renormalization group is to obtain, from the renormalization recursion relations, flow diagrams in the parameter space of the system, i.e., the space spanned by the different kinds of interactions in the Hamiltonian. The attractors (trivial fixed points) and their basins in the flow diagram correspond to regions of different thermodynamic phases. These regions are separated by critical frontiers associated with semi-stable attractors (relevant fixed points) which determine the universality class of the critical exponents. 
More unstable attractors located on the critical surfaces lead to multicritical frontiers (or, as it happens to be more usual, just multicritical points). There can also be a first-order frontier line linking a multicritical point to a first-order fixed point located at infinite values of the Hamiltonian parameters. Moreover, the singularities of the critical frontiers on the multicritical regions are characterized by the crossover exponents. So, from the above general view, the topology of flux diagrams allows one to achieve qualitative information about the critical behavior of the system, e.g., universality, order of transitions, crossover and multicriticality, among others. Regarding the real space renormalization group approach, which will be the subject of this work, one can also say that in most cases rather accurate quantitative values for the exponents and transition lines are obtained.

Just for completeness we should say that, from a different and more modern point of view, the renormalization group is essentially based on the fractal structure exhibited by the system at the critical point. A fractal, named by Mandelbrot [12], describes structures consisting of parts of any size (i.e., in any scale) which are similar to the whole. Consequently, the renormalization group commonly establishes a recursion relation transforming the parameter space of the Hamiltonian into itself, leaving thus the underlying physics unchanged. Due to this continuous scale invariance a fractional dimension is ascribed to the fractal (and, as a consequence, to the critical exponents, as we shall shortly see in this Introduction), in contrast to the integer dimension of the translationally invariant Euclidian space. There are, however, systems exhibiting discrete scale invariance (a weaker form of scale invariance symmetry which is not continuous) where dimensions or exponents are complex. Even in such cases the renormalization group formalism turns out to be quite useful [5]. The interested reader is referred to the recent review by Sornette for further details concerning discrete scale invariance and complex dimensions [13].

The renormalization group procedure has been extensively reviewed in literature (see, for instance, the excellent reviews in References [2, 3, 9, 11]; see also a more recent text by Yeomans on this subject [14]). Regarding the RSRG several techniques have been proposed and applied with success to various problems. Among the simplest ones we have decimation [15], Migdal-Kadanoff [16] and Niemeijer-van Leeuwen cells [10] approaches, which have also been discussed in the reviews referenced above. More accurate procedures include Monte Carlo renormalization group [17], cor- relation function preserving renormalization group [18] and phenomenological renormalization group [19]. It is not the scope of the present work to discuss all the methods above, rather just the phenomenological ones will be treated. We should mention excellent reviews on Monte Carlo renormalization group [20] and correlation function phenomenological renormalization group [21].

Despite its success, the ordinary RSRG has some drawbacks in the core of its implementation. Although exact results are in general achieved in classical models for dimension $d=1$, in most cases for $d>1$ some uncontrollable, and rather obscure, approximations have to be done in order to properly obtain the renormalization recursion relations. In addition, one has also to deal with the awkward "proliferation of parameters", usually encountered in approaches such as decimation [15].

One class of RSRG methods, free from the drawbacks presented above, is the so-called "phenomenological renormalization" or finite size scaling approach. In these methods one computes (exactly) thermodynamic quantities $P_{L}$ and $P_{L^{\prime}}$ for two different finite systems and, from the expected scaling relation obeyed for this quantity $P$ in the limit where $L^{\prime}(<L)$ tends to infinity, renormalization group recursion equations can be obtained. In this way the critical properties of the infinite system are obtained (approximately) from the knowledge of the corresponding properties of its finite lattice counterpart.

The finite systems used in these phenomenological renormalizations depend strongly on the geometry of the lattice. For hypercubic lattices there are two specific geometries of particular interest:

i) a finite system in all directions consisting of a hypercube of side $L$ on a $d$-dimensional lattice;

ii) a system infinite in one direction and finite in the other $d-1$ dimensions with cross-sectional area characterized by the length $L$.

Other geometries, such as systems infinite in two dimensions, can also be considered. However, due to the difficulty in obtaining exact solutions even for twodimensional models, such geometry will not be discussed here. On the other hand, boundary conditions applied to the finite systems depend on the particular renormalization group strategy. The common ones are the periodic boundary conditions and, in this case, just bulk criticality is studied. There are, however, some methods which allow to study bulk and surface criticality by taking free boundary conditions on finite lattices. 
Sometimes helical boundary conditions are imposed in geometries ii) in order to obtain a sparse transfer submatrix leaving the problem numerically more tractable [22].

To illustrate, in a quite simple way, the procedure of writing down a RSRG recursion relation for finite systems let us take, without loss of generality, the magnetic language by means of the spin-S Ising Hamiltonian model defined as

$$
\mathcal{H}=-J \sum_{<i, j\rangle} S_{i} S_{j}-h \sum_{i=1}^{\mathcal{N}} S_{i},
$$

where the first term is a sum over nearest neighbor pairs of spins $\langle i, j\rangle$ (which might be conveniently extended to more distant neighbors) located on an arbitrary lattice and the second term is a single sum over all the $\mathcal{N} \rightarrow \infty$ spins of the lattice. $J$ is the exchange interaction which is ferromagnetic for $J>0$ or antiferromagnetic for $J<0$ and $h$ is an applied external magnetic field. The spin variables $S_{i}$ take values $S_{i}=S, S-1, \ldots,-S$ where $S$ is a fixed integer or semiinteger positive number. This model exhibits a secondorder phase transition at $K=K_{c}$ and $H=0$, where $K=\beta J$ is the reduced exchange interaction, $H=\beta h$ is the reduced external magnetic field, $\beta=1 / k_{B} T$ and $k_{B}$ is the Boltzmann constant. At zero external field the correlation length $\xi$ close to the transition is expected to diverge as

$$
\xi \approx|\epsilon|^{-\nu},
$$

where $\epsilon=K-K_{c} \approx 0$ and $\nu$ is the corresponding critical exponent.

From the Kadanoff block construction [6] it is shown that close to the transition the singular part of the free energy per site $f_{s}(\epsilon, H)$ is a homogeneous function of its thermodynamic variables (as stated by Widom in 1965 [23]) i.e.,

$$
f_{s}(\epsilon, H)=\ell^{-d} f_{s}\left(\ell^{1 / \nu} \epsilon, \ell^{y_{H}} H\right),
$$

where $\ell$ is an arbitrary scaling factor, $d$ is the dimensionality of the corresponding lattice, and $y_{H}$ is the magnetic critical exponent related to the magnetization $m$ through $m=H^{1 / \delta}$, with $\delta=y_{H} /\left(1-y_{H}\right)$. Now, any other quantity $P$, obtained as derivatives of the above free energy, will behave as power laws

$$
P \approx|\epsilon|^{-\sigma},
$$

where $\sigma$ is the critical exponent of the quantity $P$. For example, the magnetization

$$
m(K, H)=\frac{\operatorname{Tr}\left(\frac{1}{\mathcal{N}} \sum_{j=1}^{\mathcal{N}} S_{j}\right) \exp (-\beta \mathcal{H})}{\operatorname{Tr} \exp (-\beta \mathcal{H})},
$$

is obtained, close to criticality, from

$$
m(\epsilon, H)=-\frac{\partial f_{s}(\epsilon, H)}{\partial H}=\ell^{-d+y_{H}} m\left(\ell^{1 / \nu} \epsilon, \ell^{y_{H}} H\right) .
$$

At $H=0$ and choosing $\ell^{-1 / \nu}=\epsilon(\epsilon>0) \mathrm{Eq}$. (6) gives, after comparing to Eq. (4), $\sigma=\beta=$ $-\left(d-y_{H}\right) \nu$. Similarly, for the zero field specific heat $C=-T\left(\partial^{2} f_{s} / \partial T^{2}\right)_{H=0}$ and zero field magnetic susceptibility $\chi=(\partial m / \partial H)_{H=0}$ it is easy to obtain $\sigma=$ $\alpha=2-d \nu$, and $\sigma=\gamma=\left(2 y_{H}-d\right) \nu$, respectively. These power laws are a signature of the continuous scale invariance of the system at criticality.

On the other hand, a finite system of linear dimension $L$ close to $K_{c}$ will of course present a non-diverging characteristic length and its correlation length will, at the maximum, be given by $\xi \approx L$. Inserting this relation in Eq. (2) and substituting for $\epsilon$ in Eq. (4) one gets

$$
P \approx L^{\sigma / \nu}=L^{\phi},
$$

where $\phi=\sigma / \nu$ is called the anomalous dimension of the quantity $P$. As $\phi$ is commonly a fractional number it is clear again from the above relation the effects of continuous scale invariance on dimensions and exponents of critical systems.

According to the finite size scaling hypothesis, the generalized scaling relation obeyed by any thermodynamic quantity $P$ taking into account the finite size $L$ of the system can be expressed as [24, 25]

$$
P(\epsilon, H, L)=\ell^{\phi} P\left(\ell^{1 / \nu} \epsilon, \ell^{y_{H}} H, \ell^{-1} L\right),
$$

where $L \rightarrow \infty$ is the linear dimension of the finite system and $\epsilon \approx 0, H \approx 0$. By identifying the right hand side of Eq. (8) to the respective quantity $P$ for a smaller system of linear dimension $L^{\prime}$ we choose the scaling factor

$$
\ell=\frac{L}{L^{\prime}}
$$

Expressing now the variables $\ell^{1 / \nu} \epsilon=\epsilon^{\prime}=K^{\prime}-K_{c}$ and $\ell^{y_{H}} H=H^{\prime}$, Eq. (8) can be rewritten as

$$
\frac{P_{L^{\prime}}\left(K^{\prime}, H^{\prime}\right)}{L^{\prime \phi}}=\frac{P_{L}(K, H)}{L^{\phi}}
$$

where the system size is now given as a subscript instead of a variable (to be more apparent) and primed quantities refer to the smaller system $L^{\prime}$. This is the basic equation from which a mapping $(K, H) \rightarrow\left(K^{\prime}, H^{\prime}\right)$ is obtained with the rescaling factor defined in Eq. (9). It is clear that the only source of inaccuracy in this relation resides in the finite values taken for $L$ and $L^{\prime}$ in 
order to get exact calculations for $P_{L}$ and $P_{L^{\prime}}$. Nevertheless, it has been noted that such approaches are applicable to rather small systems of no more than a few degrees of freedom. Of course, the bigger the finite systems, the better the results achieved, and it turns out that sometimes they exceed in accuracy more conventional methods such as series expansion, $\epsilon$-expansion or other renormalization group procedures.

Different quantities $P$ will of course generate different approaches. The first procedure, and in our opinion one with the most accurate results, was proposed by Nightingale [19] by using the correlation length computed for finite lattices consisting of infinite strips with finite width, i.e., the geometry ii) discussed above. From the basic ideas of the renormalization group theory it is well known that the transformed system with less degrees of freedom has a smaller correlation length which is the old one reduced by the rescaling factor $\ell$, namely

$$
\xi^{\prime}\left(K^{\prime}, H^{\prime}\right)=\frac{\xi(K, H)}{\ell} .
$$

From equations (9) and (11) we then get

$$
\frac{\xi^{\prime}\left(K^{\prime}, H^{\prime}\right)}{L^{\prime}}=\frac{\xi(K, H)}{L} .
$$

This means that for the correlation length the anomalous exponent $\phi$ is equal to unity and the above equation was interpreted by Nightingale as a renormalization group transformation of the infinite system from which fixed points, critical exponents, etc., are obtained. Since Eq. (12) involves just a single function, we obtain just one scalar recursion relation instead of the usual multidimensional recursion relation on a set of coupling constants such as $(K, H)$ in the present example. So, flow diagrams analyses are not possible when the Hamiltonian has more than two parameters. However, second-order transition lines and estimates of critical exponents can be obtained by determining a mapping,

$$
K \rightarrow K^{\prime}=R(K ; r)
$$

for a fixed value of $r=H / K$, where the recursion relation $R(K ; r)$ is, in principle, obtained from Eq. (12). The critical line is given by the fixed point solutions $K^{\prime}=K=K^{*}=R\left(K^{*} ; r\right)$ as a function of $r$, and estimates of the critical exponent are obtained from the linearization of $R$ around $K^{*}$,

$$
K^{\prime}-K^{*}=\lambda_{T}\left(K-K^{*}\right),
$$

where the thermal eigenvalue $\lambda_{T}$ is given by

$$
\lambda_{T}=\left.\frac{\partial K^{\prime}}{\partial K}\right|_{K^{*}}=\left.\frac{\partial R(K ; r)}{\partial K}\right|_{K^{*}},
$$

with the corresponding thermal critical exponent

$$
\ell^{1 / \nu}=\lambda_{T} .
$$

Estimates of the magnetic critical exponent are extracted from

$$
\ell^{y_{H}}=\left.\frac{\partial H^{\prime}}{\partial H}\right|_{K^{*}},
$$

from which, using Eq.(12), we have

$$
\ell^{2 y_{H}+1}=\left(\frac{\partial^{2} \xi^{\prime} / \partial H^{\prime 2}}{\left(\partial^{2} \xi / \partial H^{2}\right.}\right)_{K^{*}} .
$$

The second derivatives appear in obtaining $y_{H}$ since $\xi$ is commonly an even function of $H$. The procedure outlined above is quite general when using only one recursion relation and should be applied to any function $R(K ; r)$ (coming, for example, from other renormalization group scheme) as well as to any couplings (not necessarily an external field).

Other thermodynamic functions (such as, for instance, specific heat or magnetic susceptibility) can also be used to study the critical properties of statistical mechanical systems through Eq. (10). The main problem in these cases is that the exponent $\phi$ is in general not known. The method can, nevertheless, be implemented by force through the use of three different finite systems ( $\left.L, L^{\prime}, L^{\prime \prime}\right)$ and taking $\phi$ as the value that yields the same fixed point solution for the recursion relation (10) from $\left(L, L^{\prime}\right)$ and $\left(L^{\prime}, L^{\prime \prime}\right)$ clusters, respectively [26]. In the present work we will be concerned only with methods where $\phi$ has a known value.

By taking $P$ as the order parameter of the system and together with mean field calculations one can obtain the so-called mean field renormalization group (MFRG) approach [27]. Here, it is possible to overcome the difficulty of not knowing the order parameter anomalous dimension $\phi$ and finding out a recursion relation free from any exponent. This procedure will be discussed in more detail in the next section. It is also possible, in some models having more than one order parameter, to obtain complete flux diagrams in the Hamiltonian coupling space due to additional recursion relations. Moreover, by taking three clusters at the same time, it is allowed to study bulk and surface critical behavior since the used finite lattices must have open boundary conditions.

It is clear from Eq. (10) that in carrying out phenomenological renormalization group calculations one 
desires to choose quantities $P$ in which their anomalous dimensions are known a priori. A more recent proposal, for particular quantities having $\phi=0$, has been introduced by de Oliveira in the finite size scaling renormalization group (FSSRG) method [28, 29, 30]. This method is also capable to circumvent, in some cases, the lacking of flow diagram lines in the parameter space by considering more than one quantity at the same time and obtaining additional recursion relations, as in some versions of the previous MFRG approach, even for models presenting just one order parameter. This approach will also be discussed later on.

The purpose of this work is thus to review some phenomenological real space renormalization group procedures namely, the mean field renormalization group, the effective field renormalization group (EFRG) and the finite size scaling renormalization group. We will not discuss in detail the Nightingale phenomenological renormalization since it has already been widely discussed in literature (see, for instance, Reference [31] and references therein). Special emphasis will then be given to the MFRG since it has been extended and improved in different ways and applied to a vast class of models (classical, quantum, geometrical, pure and diluted, static and dynamic). We will also not discuss the results achieved in each of its applications. We will be mainly concerned here with all the versions of the method and its general formulations, some of them not previously published in the literature. A simple list of references in alphabetical order (without any comments) on works involving the use of MFRG up to the year 1993 has already been given by Croes and Indekeu [32].

This review is arranged as follows. In the next section we present the formalism of the mean field renormalization group for the study of static and dynamic critical phenomena. In Section III the exact results from the MFRG are presented. Section IV gives an up to date application of the method in static problems, and Section $V$ does the same concerning the dynamic critical behavior. Related phenomenological renormalization group approaches are presented in section VI and some final remarks are discussed in section VII.

\section{Phenomenological Mean field renormalization group ap- proach}

The phenomenological mean field renormalization group approach uses the order parameter as the quan- tity to be renormalized. In magnetic models it is given by the magnetization of the system which can generally be written as $m(\mathbf{K}, H)$, where the vector $\mathbf{K}$ represents all the reduced coupling constant interactions of the Hamiltonian and $H$ is the reduced external magnetic field. It means that the first sum in Eq. (1) may now not be restricted to first neighbors but may also include more distant interactions as well as additional fields. For instance, when $\mathbf{K}$ is written as $\mathbf{K}=\left(K_{1}, K_{2}, K_{3}, K_{4}, \ldots\right), K_{1}$ should represent nearest neighbor interaction, $K_{2}$ next nearest neighbor interaction, $K_{3}$ a crystal field interaction, $K_{4}$ a four spin interaction, and so on. Since the MFRG has been proposed by Indekeu, Maritan and Stella in 1982 [27] the method has been widely used in treating several statistical mechanical problems as well as frequently improved in different aspects of its formulation. This section is devoted to review its basic assumptions and discuss some new implementations.

\section{A. MFRG}

In the original MFRG one first considers two clusters of interacting spins containing $N$ and $N^{\prime}$ sites, with $N^{\prime}<N$. The surrounding spins of these clusters are fixed to a value $b$ and $b^{\prime}$, respectively, which, in a sense, can be viewed as effective magnetizations representing the remaining spins of the infinite lattice and behave as a symmetry breaking field in each cluster. The magnetizations per site $m_{N}(\mathbf{K}, H ; b)$ and $m_{N^{\prime}}\left(\mathbf{K}^{\prime}, H^{\prime} ; b^{\prime}\right)$ can exactly be computed from

$$
m_{N}(\mathbf{K}, H ; b)=\frac{\operatorname{Tr}\left(\frac{1}{N} \sum_{j=1}^{N} S_{j}\right) \exp \left(-\beta \mathcal{H}_{N}\right)}{\operatorname{Tr} \exp \left(-\beta \mathcal{H}_{N}\right)},
$$

where the trace is taken over the ensemble defined by the cluster Hamiltonian $\mathcal{H}_{N}(\mathbf{K}, H ; b)$ and $S_{j}$ is the corresponding spin operator. Similar expression holds for the smaller cluster $N^{\prime}$. The usual mean field type approximation is obtained by assuming $m_{N}(\mathbf{K}, H ; b)=b$ (or $m_{N^{\prime}}\left(\mathbf{K}^{\prime}, H^{\prime} ; b^{\prime}\right)=b^{\prime}$ ) from which $m=b\left(\right.$ or $m^{\prime}=b^{\prime}$ ) is solved self-consistently at $H=0\left(H^{\prime}=0\right)$ and the criticality is identified with the bifurcation point in the above equations. Although this mean field approximation improves the critical couplings as the cluster size gets larger, the critical exponents are always the classical ones (in particular, for the Ising model described in Eq. (1) one has $\alpha=0, \beta=1 / 2$ and $\gamma=1$ for any lattice dimension).

Instead of the naive assumption of just equalling the symmetry breaking fields to the magnetization per spin 
for each cluster, the MFRG assumes that these magnetizations are related through the scaling relation given by Eq. (6) for the order parameter of the infinite system close to criticality [10]

$$
m_{N^{\prime}}\left(\mathbf{K}^{\prime}, H^{\prime} ; b^{\prime}\right)=\ell^{d-y_{H}} m_{N}(\mathbf{K}, H ; b),
$$

where the rescaling factor $\ell$ can be defined by the usual way

$$
\ell=\left(N / N^{\prime}\right)^{1 / d}
$$

which is equivalent to definition (9) for symmetric blocks of size $N=L^{d}$. As Eq. (20) should hold for systems near the critical point, the magnetizations for each finite cluster must be very small. Such condition is achieved by letting $b \ll 1$ and $b^{\prime} \ll 1$, as well as $H \ll 1$ and $H^{\prime} \ll 1$, since any finite system has no spontaneous magnetization. Equation (20) can thus be expanded and, to lowest order in $b, b^{\prime}$ and $H, H^{\prime}$ gives

$$
g_{N^{\prime}}\left(\mathbf{K}^{\prime}\right) H^{\prime}+f_{N^{\prime}}\left(\mathbf{K}^{\prime}\right) b^{\prime}=\ell^{d-y_{H}} f_{N}(\mathbf{K}) b+\ell^{d-y_{H}} g_{N}(\mathbf{K}) H
$$

where

$$
\begin{array}{lll}
f_{N^{\prime}}\left(\mathbf{K}^{\prime}\right)=\left.\frac{\partial m_{N^{\prime}}\left(\mathbf{K}^{\prime}, H^{\prime} ; b^{\prime}\right)}{\partial b^{\prime}}\right|_{H^{\prime}=0, b^{\prime}=0} & ; & f_{N}(\mathbf{K})=\left.\frac{\partial m_{N}(\mathbf{K}, H ; b)}{\partial b}\right|_{H=0, b=0}, \\
g_{N^{\prime}}\left(\mathbf{K}^{\prime}\right)=\left.\frac{\partial m_{N^{\prime}}\left(\mathbf{K}^{\prime}, H^{\prime} ; b^{\prime}\right)}{\partial H^{\prime}}\right|_{H^{\prime}=0, b^{\prime}=0} & ; & g_{N}(\mathbf{K})=\left.\frac{\partial m_{N}(\mathbf{K}, H ; b)}{\partial H}\right|_{H=0, b=0} .
\end{array}
$$

As $b$ and $b^{\prime}$ are also viewed as magnetizations and are very small they are assumed to satisfy the same scaling relation given by Eq. (20), i.e.,

$$
b^{\prime}=\ell^{d-y_{H}} b
$$

From equations (22) and (25) one then gets

$$
f_{N^{\prime}}\left(\mathbf{K}^{\prime}\right)=f_{N}(\mathbf{K})
$$

obtaining by equalling the coefficients of $b^{\prime}$ and

$$
g_{N^{\prime}}\left(\mathbf{K}^{\prime}\right) H^{\prime}=\ell^{d-y_{H}} g_{N}(\mathbf{K}) H
$$

obtaining by equalling the external field terms. Equation (26), which is independent of any exponent, is interpreted as a recursion relation for the coupling constants $\mathbf{K}$ according to the ordinary MFRG, while equation(27), as shown below, is used to estimate the magnetic critical exponent $y_{H}$ (the recursion relation does not depend on the external field). Clearly, for a multidimensional Hamiltonian parameter space this scalar recursion relation (26) does not provide complete flux diagrams. This sole equation can, however, be used to estimate critical exponents and locate critical surfaces by considering a mapping $K_{1} \rightarrow$ $K_{1}^{\prime}=R\left(K_{1}, r_{1}, r_{2}, \ldots\right)$ for fixed values of the ratio $r_{1}=K_{2} / K_{1}, r_{2}=K_{3} / K_{1}, \ldots$, where $\mathbf{K}=\left(K_{1}, K_{2}, \ldots\right)$ and the function $\mathrm{R}$ is, in principle, obtained from the rescaling relation (26). By computing the fixed point solution $K_{1}=K_{1}^{\prime}=K_{1}^{*}=R\left(K_{1}^{*}, r_{1}, r_{2}, \ldots\right)$ as a function of $r$ one determines the critical surface, represented here as $\mathbf{K}^{*}=\left(K_{1}^{*}, r_{1}, r_{2}, \ldots\right)$, and the corresponding exponents are achieved through

$$
\nu=\frac{\ln \ell}{\ln \lambda_{T}},
$$

as stated by Eq. (16) for the correlation length critical exponent where

$$
\lambda_{T}=\left.\frac{\partial K_{1}^{\prime}}{\partial K_{1}}\right|_{\mathbf{K}^{*}}=\left.\left(\frac{\partial f_{N}}{\partial K_{1}}\left(\frac{\partial f_{N^{\prime}}}{\partial K_{1}^{\prime}}\right)^{-1}\right)\right|_{\mathbf{K}^{*}},
$$

and

$$
g_{N^{\prime}}\left(\mathbf{K}^{*}\right)=\ell^{d-2 y_{H}} g_{N}\left(\mathbf{K}^{*}\right)
$$

for the magnetic critical exponent $y_{H}$, where the relation $H^{\prime}=\ell^{y_{H}} H$ has been used in Eq. (27).

This approach has been applied to a great variety of statistical models and quite good results have been obtained even by taking the simplest choice for the clusters, namely, $N^{\prime}=1$ and $N=2$. Some exact results are also obtained from this method when treating some specific systems. A summarized discussion of such studies will be given in the next sections. However, in order to taste the simplicity of the method and its potentialities let us apply it to three different problems by taking the smallest clusters depicted in Fig. 1.

\section{spin-1/2 nearest neighbors Ising Model}

The Hamiltonians $\mathcal{H}_{1}$ and $\mathcal{H}_{2}$ for the clusters of one and two spins shown in Figs. 1(a) and 1(b) can be written as

$$
\mathcal{H}_{1}=-c J^{\prime} b^{\prime} S_{1}-h^{\prime} S_{1}
$$




$$
\mathcal{H}_{2}=-J S_{1} S_{2}-(c-1) J b\left(S_{1}+S_{2}\right)-h\left(S_{1}+S_{2}\right),
$$

where $S_{i}= \pm 1$ and $c$ is the coordination number of the lattice. From the definition of the magnetization (19) one easily has

$$
\begin{gathered}
m_{1}=\tanh \left(c K^{\prime} b^{\prime}+H^{\prime}\right), \\
m_{2}=\frac{\sinh [2(c-1) K b+2 H]}{\cosh [2(c-1) K b+2 H]+e^{-2 K}},
\end{gathered}
$$

which, for small values of $b, b^{\prime}, H$ and $H^{\prime}$ reduce to

$$
\begin{gathered}
m_{1}=c K^{\prime} b^{\prime}+H^{\prime}, \\
m_{2}=\frac{2(c-1) K b}{1+e^{-2 K}}+\frac{2 H}{1+e^{-2 K}} .
\end{gathered}
$$

The usual mean field approximation assumes $m_{1}=b^{\prime}$ and $m_{2}=b$ yielding, at zero external field, $K_{c}=1 / c$ and $1+e^{-2 K_{c}}=2(c-1) K_{c}$. One can note that from both clusters there is a (wrong) finite critical temperature $T_{c} \neq 0$ for the one-dimensional model $(c=2)$. For a future comparison to the two-dimensional model $(c=4)$ the later expressions give $K_{c}=0.250$ and $K_{c}=0.286$ for $N^{\prime}=1$ and $N=2$, respectively. Moreover, by further expanding equations (33) and (34) up to order $b^{\prime 3}$ and $b^{3}$ it is easy to show that the corresponding critical exponents take the classical values, independent of the dimensionality of the lattice.

Reminding now equations (22-27) the corresponding MFRG recursion relation

$$
c K^{\prime}=\frac{2(c-1) K}{1+e^{-2 K}}
$$

gives

$$
K_{c}=\frac{1}{2} \ln \frac{1}{c-2},
$$

where $K_{c}=K^{\prime}=K$ is the non-trivial fixed point solution of the equation (37). The above result is the same as that obtained from the Bethe approximation [33]. The critical exponents according to equations (29) and (30) are

$$
\begin{aligned}
\ell^{1 / \nu} & =1+\frac{(c-2)}{2(c-1)} \ln \frac{c}{c-2}, \\
\ell^{d-2 y_{H}} & =\frac{(c-1)}{c}, \quad \ell=2^{1 / d} .
\end{aligned}
$$

In this case, for the one-dimensional model $(c=2)$ one has the exact critical temperature $T_{c}=0$ and critical exponent $y_{H}=1$ (these values are in fact obtained for any cluster sizes), while the critical exponent $\nu=1$ is only achieved by comparing two chains with $N$ and $N-1$ spins in the limit $N \rightarrow \infty$. Numerical values for the two-dimensional model are $K_{c}=0.347, \nu=1.667$ and $y_{H}=1.415$, which are, when compared to the previous mean field results (and without further effort), closer to the exact ones, mainly for the critical coupling (see Table I). The corresponding results for the $d=3$ lattice using the present clusters are listed in Table II.

\section{Site percolation}

The site percolation [42] is a geometrical problem defined on a regular infinite lattice where each site can independently be occupied or empty with probabilities $p$ and $1-p$, respectively ( $p$ is also viewed as the concentration of the occupied sites). A cluster is formed by grouping together nearest neighbors occupied sites. At $p=1$ one has an infinite cluster linking all sites. On the other hand, for small $p$ one has an infinite number of independent finite clusters. Then there is a critical concentration $p_{c}$ above which the size of the clusters (at least one) becomes infinite. The mean linear size of the clusters is the correlation length $\xi$ which, close to the critical threshold diverges as

$$
\xi \approx\left|p-p_{c}\right|^{-\nu_{p}}
$$

where $\nu_{p}$ is the percolation correlation length critical exponent. $p_{c}$ depends on the topology of the lattice and $\nu_{p}$ depends only on the lattice dimensionality.

Let us now consider the same clusters (a) and (b) in Fig. 1 for this problem [43, 44]. The order parameter $P_{N}$ is defined by the probability of a particular site to belong to the infinite cluster. $b$ and $b^{\prime}$ will be now the effective values of the probability, for surrounding sites of each block, to belong to the infinite cluster. For the one site cluster $\left(N^{\prime}=1\right)$ its probability of belonging to the infinite cluster is $p^{\prime} b^{\prime}$ times the number $c$ of surrounding sites, i.e.

$$
P_{1}=c p^{\prime} b^{\prime} .
$$

In a similar way, the probability of any of the two sites of the $N=2$ cluster is, up to first-order in $b$, given by

$P_{2}=p(1-p)(c-1) b+2 p^{2}(c-1) b=p(1+p)(c-1) b$.

The first term on the left hand side of the above equation takes into account the contribution of the configuration where one site of the cluster is occupied and the other one is empty, and the second term the contribution when both sites are occupied. In all terms just configurations with one surrounding site occupied with probability $b$ are taken since configurations presenting two or more mean field sites will contribute to the order parameter a factor of order $b^{2}$ or greater. Mean field approximation $\left(P_{1}=b^{\prime}\right.$ and $\left.P_{2}=b\right)$ gives $p_{c}=1 / c$ for the 
smaller cluster and $p_{c}=\frac{1}{2}\left(\sqrt{\frac{c+3}{c-1}}-1\right)$ for the bigger one, while the MFRG furnishes the recursion relation

$$
p^{\prime} c=p(1+p)(c-1),
$$

from which one obtains the non trivial fixed point $p=p^{\prime}=p_{c}$

$$
p_{c}=\frac{1}{c-1},
$$

again identical to the Bethe approximation [45]. The corresponding critical exponent is given by

$$
\ell^{1 / \nu_{p}}=\frac{c+1}{c} .
$$

As in the previous case, for the one-dimensional model the mean field approximation results $p_{c}<1$, contrary to the exact value $p_{c}=1$. This exact result is however obtained from the MFRG recursion relation (44). The correct value of $\nu_{p}=1$ is achieved only in the limit of infinite chains. For the two-dimensional square lattice equations (44) and (45) furnish $p_{c}=1 / 3$ and $\nu=1.55$, which should be compared to the series values $p_{c}=0.59$ and $\nu=1.33$ [46]. The results are analogous for $d=3$. In addition, the related problem of bond percolation where bonds are independently present linking two neighboring sites with probability $p$ or absent with probability $1-p$ can similarly be treated from this formalism. It turns out that the results are identical to those above for the site problem when using the smallest clusters. Only by taking bigger finite systems the approach is able to distinguish between the site percolation and the bond percolation problems.

\section{Directed self-avoiding random walk}

Let us briefly mention the directed self-avoiding random walk since it is, in some sense, related to the site percolation problem treated above. In order to define the directed self-avoiding random walk (DSARW) let us consider a $d$-dimensional hypercubic lattice [47]. The DSARW is the geometrical object built by starting at a given point (the origin) and advancing at random through steps linking nearest neighbors sites, with the constraint that any step be only along the positive directions of the cartesian axis. Letting $w$ be the fugacity associated with a step, one has that for $w$ larger than a critical value $w_{c}$ the walk will, on average, extend to an arbitrarily large distance. In this case one can distinguish two different directions: one parallel $(\|)$ defined along the bisectrix of the angle formed by the cartesian axis, and another perpendicular $(\perp)$ taken orthogonally to the previous one. It turns out that as $w$ approaches $w_{c}$ the root mean square of the parallel and perpendicular displacements diverge as power laws of the form $\left(w-w_{c}\right)^{\nu} \|$ and $\left(w-w_{c}\right)^{\nu \perp}$, respectively, with $\nu_{\|}$and $\nu_{\perp}$ being the corresponding critical indices. Figs. 1(c) and 1(d) illustrates the smallest possible clusters which can be used by the MFRG in order to treat the DSARW on a two-dimensional lattice.

Defining now the order parameter $P_{N}$ as the generating function of all walks that begin at the origin site and end at the mean field sites (with $b$ being the effective value of this order parameter for the surrounding sites) one arrives at

$$
P_{1}=2 w^{\prime} b^{\prime}
$$

since there are two ways with probability $w^{\prime} b^{\prime}$ of linking the origin of this smallest cluster to its two boundary sites and

$$
P_{2}=4 w^{2} b,
$$

where for this cluster one has four different ways of linking the origin to the three boundary sites all having the same probability $w^{2} b$. The MFRG recursion relation is then $w^{\prime}=2 w^{2}$ from which one has the exact non trivial fixed point $w_{c}=1 / 2$. Exact values for the critical indexes (which are more laborious to be computed) $\nu_{\|}=1$ and $\nu_{\perp}=1 / 2$ are also obtained (in fact, for any cluster size, as well as for any dimension $d$. See Ref. [48] for more details).

Although in all models above the calculation of the order parameters could be readily performed for the smallest clusters, larger systems will drastically increase the analytical and computational effort. Tables I and II contain the results obtained for the spin- $1 / 2$ Ising model with nearest neighbor interactions up to the greatest size treated so far ( $L=6$ for the twodimensional model and $L=3$ for the three-dimensional one), and Table III the corresponding values for the two-dimensional site percolation problem. A more detailed discussion of the values in these tables will be given in the next subsection.

Despite its simplicity the usual MFRG treated above deserves some critics regarding the use of larger clusters. Since the symmetry breaking fields $b$ and $b^{\prime}$, in some sense, take into account the remaining of the infinite lattice, the scaling imposed for the magnetizations $m_{N^{\prime}}$ and $m_{N}$ is the relation obeyed by the infinite system order parameter (note that equation (20) comes from the Wilson renormalization group strategy). On the other hand, the final expression of the MFRG, namely Eq. (26), is independent of the symmetry breaking fields. From this equation the functions 
$f_{N}$ and $f_{N^{\prime}}$ can be thought of being quantities satisfying the finite size scaling relation (10) with anomalous dimension $\phi=0$. However, for very large clusters such relation for $f_{N}$ and $f_{N^{\prime}}$ (with $\phi=0$ ) is not true. This is most easily seen by taking the limit of $N$ and $N^{\prime}$ going to infinity. It is clear that while the computed magnetizations are bulk quantities, the surrounding effective magnetizations on each cluster behave as surface quantities and must properly scale as surface fields. In this limit Eq. (25) is no longer valid once $y_{H}$ is the bulk magnetic critical exponent. As a consequence, the procedure will not reproduce, in general, the exact results as the size of the systems tend to infinity.

Table I: Critical values for the $d=2$ Ising model on a square lattice according to the ordinary MFRG (two clusters) and SBMFRG (three clusters). The two entries for the critical indices in the SBMFRG correspond to the values given from equations (50) and (51), respectively [1].

\begin{tabular}{|c|c|c|c|c|c|}
\hline \multicolumn{6}{|l|}{ MFRG } \\
\hline$N, N^{\prime}$ & $K_{c}$ & $\nu$ & $y_{H}$ & $y_{H S}$ & $\nu y_{H}$ \\
\hline 4,1 & 0.361 & 1.45 & 1.50 & & 2.17 \\
\hline 9,4 & 0.381 & 1.28 & 1.57 & & 2.01 \\
\hline 16,9 & 0.393 & 1.22 & 1.60 & & 1.95 \\
\hline 25,16 & 0.401 & 1.19 & 1.62 & & 1.93 \\
\hline \multicolumn{6}{|l|}{ SBMFRG } \\
\hline$N, N^{\prime}, N^{\prime \prime}$ & $K_{c}$ & $\nu$ & $y_{H}$ & $y_{H S}$ & $\nu y_{H}$ \\
\hline $9,4,1$ & 0.413 & $1.23-1.37$ & $1.63-1.56$ & $0.50-0.56$ & 2.00 \\
\hline $16,9,4$ & 0.425 & $1.16-1.22$ & $1.68-1.65$ & $0.49-0.52$ & 1.95 \\
\hline $25,16,9$ & 0.430 & $1.13-1.16$ & $1.71-1.69$ & $0.49-0.51$ & 1.92 \\
\hline $36,25,16$ & 0.433 & $1.10-1.12$ & $1.73-1.72$ & $0.49-0.50$ & 1.91 \\
\hline exact [2] & 0.441 & 1 & 1.875 & $0.5[3]$ & 1.875 \\
\hline
\end{tabular}

[1]Data from Refs. [27] and [32] for hypercubic finite clusters.

[2]Refs. [34, 35].

[3]Ref. [36].

Table II. The same as Table I for the $d=3$ simple cubic Ising model. For hypercubic clusters one has $N=L^{3}$, and for rectangular prisms $N=L(L-1)^{2}$ or $N=L^{2}(L-1)$ [1].

\begin{tabular}{|c|c|c|c|c|c|}
\hline \multicolumn{6}{|l|}{ RG } \\
\hline$N, N^{\prime}$ & $K_{c}$ & $\nu$ & $y_{H}$ & $y_{H S}$ & $\nu y_{H}$ \\
\hline 2,1 & 0.203 & 1.50 & 1.89 & & 2.84 \\
\hline 4,1 & 0.207 & 1.22 & 2.00 & & 2.44 \\
\hline 9,4 & 0.212 & 1.05 & 2.08 & & 2.18 \\
\hline \multicolumn{6}{|l|}{ SBMFRG } \\
\hline$N, N^{\prime}, N^{\prime \prime}$ & $K_{e}$ & $\nu$ & $y_{H}$ & $y_{H S}$ & $\nu y_{H}$ \\
\hline $4,2,1$ & 0.212 & $1.23-1.49$ & $2.00-1.91$ & $1.03-1.12$ & 2.85 \\
\hline $8,4,2$ & 0.215 & $0.96-1.22$ & $2.15-2.00$ & $0.90-1.05$ & 2.44 \\
\hline $12,8,4$ & 0.201 & $1.32-1.03$ & $1.95-2.09$ & $0.99-0.85$ & 2.15 \\
\hline $18,12,8$ & 0.216 & $1.04-1.19$ & $2.09-2.01$ & $0.95-1.03$ & 2.39 \\
\hline $27,18,12$ & 0.217 & $0.88-0.99$ & $2.20-2.09$ & $0.86-0.97$ & 2.07 \\
\hline other methods & $0.222[2]$ & $0.63[3]$ & $2.48[3]$ & $0.8[4]$ & 1.56 \\
\hline
\end{tabular}

[1]Data from Refs. [27] and [32].

[2]Ref. [37, 38, 39, 40].

[3]Ref. [38, 39, 40, 41].

[4]Ref. [36]. 


\section{B. Surface-Bulk MFRG (SBMFRG)}

A way to handle this situation has also been proposed by Indekeu and co-workers [49]. For large clusters, except for spins at corners or edges, almost all boundary spins are subject to the same effective field which is proportional to the surrounding fixed magnetizations. According to a rigorous finite size scaling derivation these surrounding fields do not scale like a magnetization itself but like a surface field [36]. So, for $b$ and $b^{\prime}$ one has

$$
b^{\prime}=\ell^{y_{H S}} b,
$$

where $y_{H S}$ is the corresponding surface field critical exponent. One can note that now the factor $\ell^{d-y_{H}}$ is no more eliminated from equations (22) and (48) and a simple formulation, as expressed by Eq. (26), is not available. It is needed then three clusters $N$, $N^{\prime}$ and $N^{\prime \prime}$ (with $N>N^{\prime}>N^{\prime \prime}$ ), instead of two, in order to get a renormalization group transformation. In this case, from $m_{N}(\mathbf{K}, H ; b), m_{N^{\prime}}\left(\mathbf{K}^{\prime}, H^{\prime} ; b^{\prime}\right)$ and $m_{N^{\prime \prime}}\left(\mathbf{K}^{\prime \prime}, H^{\prime \prime} ; b^{\prime \prime}\right)$ one computes $f_{N}(\mathbf{K}), f_{N^{\prime}}\left(\mathbf{K}^{\prime}\right)$, $f_{N^{\prime \prime}}\left(\mathbf{K}^{\prime \prime}\right)$ and from Eq. (22) at $H=H^{\prime}=H^{\prime \prime}=0$ together with

$$
b^{\prime}=\ell_{N N^{\prime}}^{y_{H S}} b, \quad b^{\prime \prime}=\ell_{N^{\prime} N^{\prime \prime}}^{y_{H S}} b^{\prime},
$$

it is easy to obtain

$$
\begin{gathered}
f_{N^{\prime}}\left(\mathbf{K}^{\prime}\right)=\ell_{N N^{\prime}}^{d-y_{H}-y_{H S}} f_{N}(\mathbf{K}), \\
f_{N^{\prime \prime}}\left(\mathbf{K}^{\prime \prime}\right)=\ell_{N^{\prime} N^{\prime \prime}}^{d-y_{H}-y_{H S}} f_{N^{\prime}}\left(\mathbf{K}^{\prime}\right),
\end{gathered}
$$

where

$$
\ell_{N N^{\prime}}=\left(N / N^{\prime}\right)^{1 / d}, \quad \ell_{N^{\prime} N^{\prime \prime}}=\left(N^{\prime} / N^{\prime \prime}\right)^{1 / d} .
$$

Following the well-established optimization strategy [26] the exponent $d-y_{H}-y_{H S}$ is determined selfconsistently by imposing that the two different mappings with rescaling factors $\ell_{N N^{\prime}}$ and $\ell_{N^{\prime}} N^{\prime \prime}$ possess the same fixed point $\mathbf{K}=\mathbf{K}^{\prime}=\mathbf{K}^{\prime \prime}=\mathbf{K}^{*}$. This procedure allows one to obtain unambiguously the index $d-y_{H}-y_{H S}$ while two different estimates for the critical exponents $\nu, y_{H}$ and $y_{H S}$ are achieved from each of the renormalization group transformations (50) and (51) (there are in fact three different estimates since from equations (50) and (51) one can construct an additional relation between $f_{N}{ }^{\prime \prime}$ and $f_{N}$ ).

At this point it is worthwhile to see a quantitative and comparative application of these methods in the well known control system square two-dimensional Ising model. Table I shows the critical temperature and critical exponents for different growing finite size square clusters. It is clear that a better accuracy is achieved from SBMFRG as compared to the usual
MFRG, mainly for larger clusters, where the approach to the exact results is rather apparent. However, concerning small clusters, the distinction of both methods is not so profound. First, in assumption (26) the ordinary MFRG assumes a zero value for the critical index $d-y_{H}-y_{H S}$ (the exact one being -0.375 ). From the SBMFRG one gets the best value $d-y_{H}-y_{H S}=-0.217$ for the biggest systems while for the simplest choice $N=4, N^{\prime}=2$ and $N^{\prime \prime}=1$ one has -0.124 [49], which is a quite small value. Second, for small clusters there is no even a clear distinction between surface and bulk quantities. This means that the fact of the SBMFRG being exact when $N, N^{\prime}$ and $N^{\prime \prime} \rightarrow \infty$ does not make it more correct than the original MFRG for small systems. Which method should be applied to a particular problem must be decided on practical grounds. Table II lists the results for the $d=3$ Ising model and Table III the values for the $d=2$ site percolation problem.

Table III. The same as Table I for the critical values of the $d=2$ site percolation problem.

\begin{tabular}{cccc} 
MFRG & \multicolumn{3}{c}{} \\
\hline$N, N^{\prime}$ & $p_{c}$ & $\nu_{p}$ & $y_{H S}^{p}$ \\
\hline 2,1 & $1 / 3$ & 1.56 & \\
4,2 & 0.427 & 1.52 & \\
9,4 & 0.443 & 1.47 & \\
16,9 & 0.473 & 1.43 & \\
\hline SBMFRG & \multicolumn{4}{c}{} \\
\hline$N, N^{\prime}, N^{\prime \prime}$ & $p_{c}$ & $\nu_{p}$ & $y_{H S}^{p}$ \\
\hline $9,4,1$ & 0.602 & $1.25-1.35$ & $0.72-0.69$ \\
$16,9,4$ & 0.585 & $1.35-1.35$ & $0.66-0.65$ \\
\hline other methods & $0.593[1]$ & $1.33[2]$ & - \\
\hline
\end{tabular}

[1]Ref. [42].

[2]Ref. [46].

Another point deserving some discussion concerns the definition of the rescaling factor $\ell$. As pointed out by Slotte [50] the rescaling factors given by equations (21) and (52) are, to some extent, arbitrary. Different definitions strongly affect the results of the critical exponents for small clusters. Slotte suggested a definition of the rescaling factor where the length of the cluster is measured by the number of interactions, including those with the surrounding mean field sites, instead of just the effective number of sites, i.e.,

$$
L=\left(\frac{1}{d} \sum_{i} L_{i}^{-2}\right)^{-\frac{1}{2}},
$$


where the sum runs over cartesian directions and $L_{i}$ are the number of bonds along each direction. The one spin cluster has then $L=2$ and the two-spin cluster $L=6 \sqrt{d /(9 d-5)}$. Such definition improves substantially the critical exponents [50]. There are, however, in the original MFRG, some quantities that are independent of $\ell$. One of them is clearly the fixed point $\mathbf{K}^{*}$, as can be seen from Eq. (26). The other one is the product $\nu y_{H}$. By abandoning the definition given by Eq. (21) the relation (30) can be rewritten as

$$
N^{\prime} g_{N^{\prime}}\left(\mathbf{K}^{*}\right)=\ell^{-2 y_{H}} N g_{N}\left(\mathbf{K}^{*}\right),
$$

which gives

$$
y_{H}=\frac{1}{2} \frac{\ln N g_{N}\left(\mathbf{K}^{*}\right) / N^{\prime} g_{N^{\prime}}\left(\mathbf{K}^{*}\right)}{\ln \ell},
$$

where $\ell^{d}$ in $(30)$ has been replaced by $N / N^{\prime}$. While the exponents $\nu$ and $y_{H}$ separately depend on the rescaling factor, it is easy to see now from equations (28) and (55) that the product $\nu y_{H}$ is independent of $\ell$, removing thus a principal weakness of the MFRG. In the unified SBMFRG this is no longer strictly true, but should still hold to a first approximation. It is then expected that the critical exponent product $\nu y_{H}$ will present a better precision than the exponents themselves. Indeed, from the last column of Table I one can see that the best product $\nu y_{H}$ from SBMFRG is within $1.8 \%$ of error from its exact value, whereas the error in critical exponents ranges between $7.8 \%-9.4 \%$. The figures for MFRG are, respectively, $3 \%$ and $14 \%-16 \%$. Roughly the same behavior is noticed in the data of Table II for the $d=3$ Ising model.

Table IV. Critical values for the $d=2$ Ising model on a square lattice according to the SBMFRG including the corner magnetic exponent $y_{H C}[1]$.

\begin{tabular}{ccccc}
$N, N^{\prime}, N^{\prime \prime}$ & $K_{c}$ & $\nu$ & $y_{H}$ & $-y_{H C}$ \\
\hline $16,9,4$ & 0.469 & $1.08-1.13$ & $1.78-1.72$ & $0.57-0.51$ \\
$25,16,9$ & 0.460 & $1.07-1.10$ & $1.80-1.76$ & $0.63-0.59$ \\
$36,25,16$ & 0.454 & $1.06-1.07$ & $1.81-1.78$ & $0.68-0.65$ \\
\hline exact [2] & 0.441 & 1 & 1.875 & $1[3]$ \\
\hline
\end{tabular}

[1]Data from Ref. [32] applying clusters in which the corner exponent can be defined.

[2]Ref. [34, 35].

[3]Ref. [52].

Similar results are also obtained in an extended version of the present approach by including surface and corner critical exponents in two-dimensional models [51]. In such $d=2$ systems the distinction between surface and corner fields is easily made. All one has to do is to include an additional corner field critical exponent $y_{H C}$ in the formalism above. Table IV quotes the results from Croes and Indekeu [32] for the twodimensional square Ising model. In this reference one also finds critical values for other two-dimensional lattices namely, triangular, honeycomb, hexagonal as well as the square lattice with next nearest neighbors interactions. On the other hand, additional edge fields will be present in three-dimensional finite lattices. However, bigger finite systems will require much computational effort and inclusion of edge fields would not be so relevant in rather small systems.

\section{Extended MFRG (EMFRG)}

In the previous subsections just Hamiltonian models with one parameter have been discussed where the trivial one-dimensional renormalization group flux is obtained. Let us now turn our attention to the case where the Hamiltonian presents more than one parameter (or coupling constant). It is clear that in this case the complete renormalization flow diagram is not defined through just the one-dimensional recursion relation as given by Eq. (26). In the particular two-dimensional case where we have $\mathbf{K}=\left(K_{1}, K_{2}\right)$, which is the most studied in literature and will be treated in this section, estimates of critical lines and critical exponents for models presenting only one order-parameter are often obtained through the mapping $K_{1} \rightarrow K_{1}^{\prime}=R\left(K_{1}, r\right)$, for fixed values of the ratio $r=K_{2} / K_{1}$, as has already been discussed in the Introduction. That is what one finds, for instance, in the random Ising model or the transverse Ising model. However, depending on the system we are studying, other additional patterns may occur where the above simple scheme cannot be applied for a two-fold coupling space (in some models even for a one order-parameter system). In these cases the thermodynamic quantities should be considered in a proper way. This subsection is devoted to present a detailed discussion of what has been done in overcoming such debility of the method as well as to present an attempt to unify the approaches done so far by using the MFRG. The formalism is intended to be quite general. However, whenever possible, some reference to particular models (without presenting results; the corresponding reference will be listed in section IV) exhibiting the behavior under consideration will be made just for clarity and as a matter of example. 


\section{one order-parameter}

For systems like, for example, the antiferromagnetic spin-1/2 Ising model in an external field (recall Eq. (1))

$$
\mathcal{H}=J \sum_{<i, j>} S_{i} S_{j}-h \sum_{i} S_{i}, \quad S_{i}= \pm 1,
$$

where $K_{1}=\beta J$ and $K_{2}=\beta h$ and the spin-1 BlumeCapel $[53,54]$ model

$$
\mathcal{H}=-J \sum_{<i, j>} S_{i} S_{j}-\Delta \sum_{i} S_{i}^{2}, \quad S_{i}= \pm 1,0
$$

where $K_{1}=\beta J$ and $K_{2}=\beta \Delta$ (the latter interaction being the crystal field anisotropy), one has two couplings $\mathbf{K}=\left(K_{1}, K_{2}\right)$ and just one order-parameter, namely, the difference $m_{+}-m_{-}$of the sublattice magnetizations $m_{+}$and $m_{-}$in the former case, and the mean value of the spin $\left\langle S_{i}\right\rangle$ at any site of the lattice in the later model. Thus, for a finite cluster of $N$ sites one can compute the order-parameter $O_{1}=O_{1}(\mathbf{K}, b, q)$ and the other non-critical variable $O_{2}=O_{2}(\mathbf{K}, b, q)$ (in the above examples $O_{2}$ is the net magnetization $m_{+}+m_{-}$of the antiferromagnet and the mean value of the square of the spin $\left\langle S_{i}^{2}\right\rangle$ in the Blume-Capel model). $b$ is the field conjugated to the order-parameter $O_{1}$ and $q$ the respective field conjugated to the noncritical variable $O_{2}$. Close to the transition just $O_{1}$ is small so the expressions above are expanded for small $b$ to give

$$
\begin{gathered}
O_{1}=F_{1}(\mathbf{K}, q) b \\
O_{2}=O_{2}(\mathbf{K}, b=0, q)=F_{2}(\mathbf{K}, q) .
\end{gathered}
$$

Note that as $b \rightarrow 0$ one has $O_{1} \rightarrow 0$ and $O_{2} \neq 0$, irrespective of the value of $q \neq 0$, whereas $O_{2} \rightarrow 0$ when one further has $q \rightarrow 0$. Similarly, for a smaller cluster of $N^{\prime}$ sites one finds

$$
\begin{gathered}
O_{1}^{\prime}=F_{1}^{\prime}\left(\mathbf{K}^{\prime}, q^{\prime}\right) b^{\prime} \\
O_{2}^{\prime}=O_{2}^{\prime}\left(\mathbf{K}^{\prime}, b^{\prime}=0, q^{\prime}\right)=F_{2}^{\prime}\left(\mathbf{K}^{\prime}, q^{\prime}\right)
\end{gathered}
$$

From now on we will omit the subscripts $N$ and $N^{\prime}$ and just denote the corresponding thermodynamic functions for different clusters by unprimed and primed quantities, respectively. According to the ordinary MFRG one then finds

$$
F_{1}^{\prime}\left(\mathbf{K}^{\prime}, q^{\prime}\right)=F_{1}(\mathbf{K}, q)
$$

which, although independent of any exponent and rescaling factor, depends on the additional fields $q$ and $q^{\prime}$. It should be said that the above behavior is not quite general. Counter examples are, among others, disordered systems [55, 56] and some quantum spin models
[56] where, despite having a two parameter Hamiltonian, they present no non-critical variables being unnecessary the introduction of extra fields $q$ and $q^{\prime}$. As a result, Eq. (62) is just dependent on $\mathbf{K}$ and $\mathbf{K}^{\prime}$ and the procedure $K_{1} \rightarrow K_{1}^{\prime}=R\left(K_{1}, r\right)$, for fixed values of the ratio $r=K_{2} / K_{1}$, outlined in the preamble of this subsection can easily be implemented.

In studying the antiferromagnetic Ising model in the triangular lattice Slotte [57] has proposed the mean field Ansatz where $q$ and $q^{\prime}$ are self consistently obtained by requiring that $\mathrm{O}_{2}^{\prime}=q^{\prime}$ and $\mathrm{O}_{2}=q$. Reasonable results, specially for the value of $K_{c}$ have been achieved. This approach has also been used in the study of the Blume-Capel model [58] where results, not consistent with the expected ones (mainly by taking the smallest cluster sizes) have been obtained, including an unexpected phase transition at a finite critical temperature for the one-dimensional version (as seen in section II.A the exact result is expected to be obtained from smallest clusters at least for the one-dimensional model).

A different choice, and equally natural, for the size dependence of the non-critical variable has been proposed by Plascak and Sá Barreto [59] and successfully applied to the Ashkin-Teller model. As this variable has its own size dependence which is not governed by finite size scaling at the transition it is assumed a renormalization group Ansatz in a way that $q^{\prime}=q, \mathrm{O}_{2}^{\prime}=\mathrm{O}_{2}$, or

$$
F_{2}^{\prime}\left(\mathbf{K}^{\prime}, q\right)=F_{2}(\mathbf{K}, q)
$$

from which $q=q^{\prime}$ is obtained as a function of $\mathbf{K}$ and $\mathbf{K}^{\prime}$ and inserted in Eq. (62). This renormalization group Ansatz has been proved to be better than the mean field one for the antiferromagnetic Ising model[60] and has been subsequently used in treating non-critical variables such as in the random Potts model [61]. Concerning the Blume-Capel model defined in Eq. (57), this improved formalism provides now exact results for its one-dimensional version and better values for the critical exponents and critical temperatures in dimensions $d>1$ [62] when compared to those from the previous usual application of the method [58]. Besides, for the particular spin-1/2 case, the above formalism reproduces the early results of the Ising model since $O_{2}=<S_{i}^{2}>=O_{1}=1 / 4$ is automatically satisfied.

The renormalization group Ansatz allows one to obtain, in addition, an unambiguous estimate for the noncritical thermodynamic function $\mathrm{O}_{2}$ at criticality (note that the mean field Ansatz gives two different estimates $\mathrm{O}_{2}=q$ and $\mathrm{O}_{2}^{\prime}=q^{\prime}$, respectively). However, it is not free from extracting results only in plausible invariant subsets given by fixed ratios $r=K_{2} / K_{1}$. So, complete analysis of the flux diagram is still not possible in such 
models.

\section{two uncoupled order-parameters}

Some systems present two different and independent order-parameters which can be used to go beyond the limitation (lacking of flux diagram) of the usual MFRG discussed so far. For example, in the symmetric Ashkin-Teller model [63] where two different spin variables exist on each lattice site, it is possible to identify two order-parameters in the problem. In a similar way, for the two-dimensional Ising model with crossing bonds one can select appropriate pairs of orderparameters depending on the region of the parameter space. In such models, for a finite block of $N$ spins, one has $O_{1}=O_{1}\left(\mathbf{K}, b_{1}\right)$ and $O_{2}=O_{2}\left(\mathbf{K}, b_{2}\right)$, where $b_{1}$ and $b_{2}$ are the symmetry breaking fields related to the order-parameters $O_{1}$ and $O_{2}$, respectively. Since for the uncoupled order-parameters $O_{1} \rightarrow 0$ when $b_{1} \rightarrow 0$ (irrespective of the value of $b_{2}$ ) and $O_{2} \rightarrow 0$ when $b_{2} \rightarrow 0$ (irrespective of the value of $b_{1}$ ) one has, to leading order in the $b^{\prime} s$,

$$
O_{1}=F_{1}(\mathbf{K}) b_{1}, \quad O_{2}=F_{2}(\mathbf{K}) b_{2} .
$$

By doing the same calculations for a smaller block of $N^{\prime}$ sites and assuming that both order-parameters are described by the same critical exponent (which is usually the case) one gets

$$
\begin{aligned}
& F_{1}^{\prime}\left(\mathbf{K}^{\prime}\right)=F_{1}(\mathbf{K}), \\
& F_{2}^{\prime}\left(\mathbf{K}^{\prime}\right)=F_{2}(\mathbf{K}) .
\end{aligned}
$$

So, contrary to the procedure prescribed in Eqs. (13)(18), in this case from equations (65) and (66) one can obtain renormalization group flux diagrams of the usual type, furnishing isolated fixed points, critical exponents, universality classes, etc. Quite good results (including some exact values at some special points in the phase diagrams) have been obtained in the Ashkin-Teller model [64] and in the two-dimensional Ising model with crossing bonds [65] through the above scheme.

\section{two coupled order-parameters - EMFRG}

The independence of the order-parameters, as stated in Eq. (64), is not commonly verified and there are some models where they do not behave in such a simple form as discussed above. In the most general case one may have $O_{1}=O_{1}\left(\mathbf{K}, b_{1}, b_{2}\right)$ and $O_{2}=O_{2}\left(\mathbf{K}, b_{1}, b_{2}\right)$ so that, to leading order in $b_{1}$ and $b_{2}$, one gets

$$
O_{1}=F_{11}(\mathbf{K}) b_{1}+F_{12}(\mathbf{K}) b_{2},
$$

$$
O_{2}=F_{21}(\mathbf{K}) b_{1}+F_{22}(\mathbf{K}) b_{2},
$$

where both order-parameters depend on $b_{1}$ and $b_{2}$. This general problem can be tackled by defining the vectors $\mathbf{O}=\left(\begin{array}{l}O_{1} \\ O_{2}\end{array}\right)$ and $\mathbf{b}=\left(\begin{array}{l}b_{1} \\ b_{2}\end{array}\right)$ so that equations $(67)$ and $(68)$ can be written as

$$
\mathrm{O}=\mathbf{F b}
$$

where the matrix $\mathbf{F}$ is given by

$$
\mathbf{F}=\left(\begin{array}{ll}
F_{11} & F_{12} \\
F_{21} & F_{22}
\end{array}\right)
$$

Similar expressions are obtained by considering a finite system $N^{\prime}$ with less degrees of freedom. It is now easy to see that when the two order-parameters have the same critical exponents the corresponding vectors $\mathrm{O}$ and $\mathrm{O}^{\prime}$ satisfy the scaling relation

$$
\mathbf{O}^{\prime}=L^{d-y_{H}} \mathbf{O},
$$

and the same for the symmetry breaking field vectors

$$
\mathbf{b}^{\prime}=L^{d-y_{H}} \mathbf{b}
$$

if we take the usual MFRG. As a result, one ends up with the following matricial equation

$$
\left(\mathbf{F}^{\prime}-\mathbf{F}\right) \mathbf{b}^{\prime}=0 .
$$

The above matricial approach is a natural extension to the general problem of two-fold Hamiltonian parameters. This extended mean field renormalization group (EMFRG) reproduces the previous cases as well as another particular approach proposed more recently by Likos and Maritan [66]. Moreover, it can give the correct Bethe limit by using the smallest clusters in treating the mixed spin Ising model (an early MFRG study of the mixed spin-1/2-spin-1 [67] Ising model has failed to obtain the corresponding Bethe results due to a different mean field assumption in order to decouple the order parameters). Some procedures in treating of the matricial Eq. (73) are now in order:

(i) The simplest solution of Eq. (73) is given by

$$
\operatorname{det}\left(\mathbf{F}^{\prime}-\mathbf{F}\right)=0 .
$$

From the above equation we do not have flow diagrams and the critical lines are obtained only for fixed values of $r=K_{2} / K_{1}$. This is a generalization of the corresponding one-order parameter where one has just $F^{\prime}\left(\mathbf{K}^{\prime}\right)=F(\mathbf{K})$. Now, by using the computed expressions of reference [67] for the two order-parameters of the mixed spin-1/2-spin-1 Ising model one obtains the 
corresponding equation (74) which gives the same critical temperature from the Bethe approximation by using the clusters depicted in Fig.1(a) and (b), as expected.

(ii) If the two parameters are decoupled one has $F_{i j}=0, i \neq j$ for any cluster and another solution for Eq. (73) can be given by equating to zero all the matrix elements of $\mathbf{F}^{\prime}-\mathbf{F}$, i.e.,

$$
F_{11}^{\prime}-F_{11}=0 \text { and } F_{22}^{\prime}-F_{22}=0
$$

which is the same result given by equations (65) and (66).

(iii) For the types of models considered by Likos and Maritan [66, 68] (in particular, models defined on a bipartite lattice with only two different ground states, a ferromagnetic and an antiferromagnetic one, separated by a stable borderline) one has $F_{11}=F_{22}$ and $F_{12}=F_{21}$ for any finite system. The last two relations reflect the additional symmetry $O_{1}\left(\mathbf{K}, b_{1}, b_{2}\right)=O_{2}\left(\mathbf{K}, b_{2}, b_{1}\right)$ exhibited by these order parameters. So, by equalling again to zero all the matrix elements of $\mathbf{F}^{\prime}-\mathbf{F}$ one gets

$$
F_{11}^{\prime}-F_{11}=0 \text { and } F_{12}^{\prime}-F_{12}=0,
$$

which are the same expressions as those proposed in reference [66] in a different context.

One can thus see that the present matricial EMFRG is capable to generalize all the previous isolated treatments done on two-dimensional parameter Hamiltonians. One can also notice that:

(a) in the general case where all the matrix elements of $\mathbf{F}$ are different from each other, Eq. (73) can furnish four distinct recursion relations, i.e., at first sight more equations than parameters. However, it should be possible, in these cases, to augment the original Hamiltonian by a suitably chosen number of interactions until the number of couplings matches the number of recursion relations. From the flow diagram so obtained in this enlarged space one could get the corresponding flows in the original restricted domain by taking the appropriate subspace in the complete Hamiltonian space.

(b) in addition, the present formalism can also be straightforwardly applied to models having more than two order-parameters simply by computing the dimensional enhanced vectors $\mathbf{O}$ and $\mathbf{b}$ and the corresponding matrix $\mathbf{F}$ for any finite cluster.

Unfortunately, the extensions ( $a$ ) and $(b)$ above have not yet been tested in studying statistical mechanics systems from MFRG and one can not say, a priori, that this approach will succeed in giving the correct critical behavior in these cases, as is usual in ordinary real space renormalization group procedures.

\section{Dynamic MFRG (DMFRG)}

So far, just equilibrium static properties have been treated through MFRG. In this subsection we discuss the extension of the mean field renormalization group ideas to non-equilibrium phenomena. Despite the simplicity of the formalism, the MFRG has been applied to a very few number of systems out of the thermodynamic equilibrium. It was employed for the first time by Indekeu, Stella and Zhang [69] to study the dynamics of the kinetic Ising model with single spin-flip Glauber transitions [70] near the equilibrium states. It has also been used to treat the dynamic critical properties of quantum spin systems [71]. As the extensions to treat the dynamics of quantum models is (at least in principle) straightforward to be done, we present below just the formalism for treating kinetic classical models with special emphasis to the Ising model by considering larger clusters than in the original work of Indekeu et al [69]. Extensions to other problems are rather easy to be performed and will be summarised in section $\mathrm{V}$.

In the dynamic approach of the MFRG one starts from the scaling relation of the magnetization, close to equilibrium and criticality. For sufficiently long times $t$ we expect the magnetization of the infinite system to scale as [72]

$$
m(\epsilon, H, t)=\ell^{-d+y_{H}} m\left(\ell^{1 / \nu} \epsilon, \ell^{y_{H}} H, \ell^{-z} t\right),
$$

which is a generalization of Eq. (6) where $z$ is the corresponding dynamic critical exponent.

Let $\mathcal{S}=\left(S_{1}, S_{2}, \ldots, S_{i}, \ldots, S_{N}\right)$, with $S_{j}= \pm 1$, represent a state of the finite system with $N$ spins, and $P(\mathcal{S}, t)$ the probability of finding the system in the state $\mathcal{S}$ at instant $t$. The time evolution of $P(\mathcal{S}, t)$ is given by the master equation

$$
\frac{d P(\mathcal{S}, t)}{d t}=\sum_{i=1}^{N}\left[P\left(\mathcal{S}^{i}, t\right) W_{i}\left(\mathcal{S}^{i}\right)-P(\mathcal{S}, t) W_{i}(\mathcal{S})\right],
$$

where $W_{i}(\mathcal{S})$ is the transition rate, per unit time, to flip the spin $S_{i}$, and $\mathcal{S}^{i}=\left(S_{1}, S_{2}, \ldots,-S_{i}, \ldots, S_{N}\right)$. The first sum in the above equation takes into account all transitions to the state $\mathcal{S}$ and the second sum all the transitions out from the state $\mathcal{S}$. If $f(\mathcal{S})$ is a given function of state $\mathcal{S}$, we can evaluate its average value by

$$
<f(\mathcal{S})>=\sum_{\mathcal{S}^{\prime}} f\left(\mathcal{S}^{\prime}\right) P\left(\mathcal{S}^{\prime}, t\right),
$$


and its time evolution through the expression

$$
\frac{d<f(\mathcal{S})>}{d t}=\sum_{i=1}^{N}<\left[f\left(\mathcal{S}^{i}\right)-f(\mathcal{S})\right] W_{i}(\mathcal{S})>.
$$

By taking the function of state as being the local magnetization of the spin $i$, we can easily write

$$
\frac{d<S_{i}>}{d t}=-2<S_{i} W_{i}(\mathcal{S})>.
$$

Let $m_{N}(t)$ and $m_{N^{\prime}}\left(t^{\prime}\right)$ be the time dependent magnetizations of two finite clusters with $\mathrm{N}$ and $N^{\prime}$ spins, respectively, i.e.,

$$
m_{N}(t)=\frac{1}{N} \sum_{i=1}^{N}<S_{i}>
$$

$$
\left.m_{N^{\prime}}(t)=\frac{1}{N^{\prime}} \sum_{i=1}^{N^{\prime}}<S_{i}^{\prime}\right\rangle,
$$

where the time dependence comes from $\left\langle S_{i}\right\rangle$ through Eq. (81). Following the original idea of the MFRG, each spin $S_{i}$ of the border of the cluster couples with an infinitesimal symmetry breaking field $b(t)$. The equations of motion for $m_{N}(t)$ and $m_{N^{\prime}}\left(t^{\prime}\right)$ are computed from Eqs. (79)-(81) by using [70]

$$
W_{i}(\mathcal{S})=\frac{1}{2}\left[1-S_{i} \tanh \left(K \sum_{j \neq i} S_{j}+c_{i} K b_{N}\right)\right],
$$

where $c_{i}$ is the number of first neighbors of $S_{i}$, which are external to the cluster, and

$$
\begin{aligned}
P_{N}(\mathcal{S}, t)= & \frac{1}{2^{N}}\left(1+\sum_{i} m_{i}(t) S_{i}+\sum_{\substack{i, i \\
i \neq j}} p_{i j}(t) S_{i} S_{j}+\right. \\
& \left.\sum_{\substack{i, j, k \\
i \neq j \neq k}} q_{i j k}(t) S_{i} S_{j} S_{k}+\ldots\right),
\end{aligned}
$$

where $m_{i}(t)=<S_{i}>, p_{i j}(t)=<S_{i} S_{j}>, q_{i j k}(t)=<$ $S_{i} S_{j} S_{k}>$, etc. The transition rate $W_{i}(\mathcal{S})$ is chosen to satisfy the detailed balance condition $P\left(\mathcal{S}^{i}, t\right) W_{i}\left(\mathcal{S}^{i}\right)=$ $P(\mathcal{S}, t) W_{i}(\mathcal{S})$ in the limit of $t \rightarrow \infty$. This is a sufficient condition to the equilibrium be attained, i.e., $d P(\mathcal{S}, t) / d t=0$ as $t \rightarrow \infty$ with the expected probability distribution $P_{N}(\mathcal{S}, t \rightarrow \infty) \propto e^{-\beta E(\mathcal{S})}$, where $E(\mathcal{S})$ is the energy of the state $\mathcal{S}$. The expression for the probability $P_{N}(\mathcal{S}, t)$, Eq. $(85)$, is an exact one and can be worked out just for rather small systems.

In general, for small clusters at $H=0$ and linearized in $b_{N}(t)$ and $b_{N^{\prime}}\left(t^{\prime}\right)$ the time derivatives of the order parameters can be written as

$$
\begin{aligned}
\frac{d m_{N}(t)}{d t} & =-A_{N}(K) m_{N}(t)+B_{N}(K) b_{N}(t), \quad(86) \\
\frac{d m_{N^{\prime}}\left(t^{\prime}\right)}{d t^{\prime}} & =-A_{N^{\prime}}\left(K^{\prime}\right) m_{N^{\prime}}\left(t^{\prime}\right)+B_{N^{\prime}}\left(K^{\prime}\right) b_{N^{\prime}}\left(t^{\prime}\right),
\end{aligned}
$$

where the expressions for $A_{N}(K), A_{N^{\prime}}\left(K^{\prime}\right), B_{N}(K)$ and $B_{N^{\prime}}\left(K^{\prime}\right)$ are computed from Eqs. (79)-(85).

According to the strategy of MFRG, we impose now the following scaling relations for the magnetizations and symmetry breaking fields:

$m_{N^{\prime}}\left(K^{\prime}, 0, \ell^{-z} t ; b_{N^{\prime}}\left(\ell^{-z} t\right)\right)=\ell^{d-y_{H}} m_{N}\left(K, 0, t ; b_{N}(t)\right)$,

and

$$
b_{N^{\prime}}\left(\ell^{-z} t\right)=\ell^{d-y_{H}} b_{N}(t),
$$

where $\ell=\left(\frac{N}{N^{\prime}}\right)^{\frac{1}{d}}$ and $t^{\prime}=\ell^{-z} t$ with $N^{\prime}<N$.

Taking the derivative of Eq. (88) with respect to $t$, and using Eqs. (86), (87) and (89), we arrive at the following recursion relations for the $A^{\prime} s$ and $B^{\prime} s$ coefficients:

$$
\begin{aligned}
& A_{N^{\prime}}\left(K^{\prime}\right)=\ell^{z} A_{N}\left(K^{\prime}\right), \\
& B_{N^{\prime}}\left(K^{\prime}\right)=\ell^{z} B_{N}(K) .
\end{aligned}
$$

The solution of the above system of equations provides the non-trivial fixed point $\left(K=K^{\prime}=K_{c}\right.$ ) and furnishes also the value of the dynamic critical exponent $z$.

As a simple example, let us take the one and two spin clusters depicted in Fig. 1(a) an (b) for this dynamic treatment in the $d$-dimensional Ising model. We have then 


$$
\begin{array}{r}
\frac{d m_{1}(t)}{d t}=-2 \sum_{\left\{S_{1}\right\}} S_{1} \frac{1}{2}\left[1-S_{1} \tanh \left(c K^{\prime} b_{1}\right)\right] \frac{1}{2}\left[1+m_{1} S_{1}\right], \\
\frac{d m_{2}(t)}{d t}=-2 \sum_{\left\{S_{1}, S_{2}\right\}} S_{1} \frac{1}{2}\left[1-S_{1} \tanh \left(K S_{2}+(c-1) K b_{2}\right)\right] \times \\
\quad \times \frac{1}{4}\left[1+m_{2}(t) S_{1}+m_{2}(t) S_{2}+p_{12}(t) S_{1} S_{2}\right],
\end{array}
$$

where in the latter relation we have used the fact that both spins in the two site cluster of Fig. 1(b) have the same time dependence. Performing the sum over the corresponding states in the two relations above one gets

$$
\begin{gathered}
\frac{d m_{1}(t)}{d t}=-m_{1}(t)+c K^{\prime} b_{1}(t), \\
\frac{d m_{2}(t)}{d t}=-(1-\tanh K) m_{2}(t)+(c-1) K\left(1-\tanh ^{2} K\right) b_{2}(t),
\end{gathered}
$$

from which one has

$$
\begin{aligned}
1 & =\ell^{z}(1-\tanh K), \\
c K^{\prime} & =\ell^{z}(c-1) K\left(1-\tanh ^{2} K\right) .
\end{aligned}
$$

By taking the ratio of Eq. (97) to Eq. (96) it is easy to see that the non-trivial fixed point and the related thermal critical exponent are the same as those obtained in the static procedure of section IIA (as well as the magnetic critical exponent $y_{H}$ if one includes the external field $H$ ), as expected. Estimates of the corresponding dynamic critical exponent $z$ are also readily achieved from above equations (see Table $\mathrm{V}$ ).

Table V. Critical temperature and dynamic critical exponent for the Ising model according to the DMFRG.

\begin{tabular}{||c|c|c||c|c|c||}
\hline \multicolumn{2}{||l|}{$\mathrm{d}=2$} & \multicolumn{2}{|c|}{$\mathrm{d}=3$} \\
\hline$N, N^{\prime}$ & $K_{c}$ & $\mathrm{z}$ & $N, N^{\prime}$ & $K_{c}$ & $\mathrm{z}$ \\
\hline 2,1 & 0.347 & 1.17 & 2,1 & 0.203 & 0.97 \\
4,1 & 0.361 & 1.39 & 4,2 & 0.207 & 1.15 \\
4,2 & 0.370 & 1.60 & 8,2 & 0.207 & 1.32 \\
9,4 & 0.381 & 2.13 & 8,4 & 0.209 & 1.49 \\
\hline & $0.441[1]$ & $2.2 \pm 0.2[2]$ & & $0.222[3]$ & $2.0[4]$ \\
\hline
\end{tabular}

[1]Refs. [34, 35].

[2]Ref. [73].

[3]Ref. [37, 38, 39, 40].

[4]Ref. [74].

In Table $\mathrm{V}$ we show the results obtained in the study of the Ising model for clusters of up to 9 spins in two dimensions (a cluster with non-equivalent boundary sites which has not been previously considered in the dynamic treatment of the model) and with up to 8 spins in three dimensions. One can see from this Table that the results are consistent with those from other approaches. However, some difficulties arise in considering bigger clusters in the present case:

(a) when we consider a cluster with four spins, by using Eq. (85) it appears correlations between pairs of spins which are located at the diagonals of the cluster;

(b) we must also be careful in the calculation of the mean values $\left\langle S_{i}\right\rangle$, because for clusters with more than four spins they are not all equivalent. It should 
be stressed that this non-equivalence among spins inside the finite cluster for $N>4$ in two-dimensions (and $N>8$ in three-dimensions) implies in the necessity to diagonalize a system of equations in order to obtain an expression like that of Eq.(86), since in this case $m_{N}(t)$ is no longer an eigenmode of the dynamics but is coupled to higher spin correlations;

(c) besides, another great difficulty arises when we try to increase the size of the clusters in this non-equilibrium approach of the MFRG. Although the method does not produce couplings of longer range in space, it is necessary to take account of the higher than two spin correlations, which naturally appear for bigger clusters. Then, we need to use methods of equilibrium statistical mechanics to evaluate these higher order correlations, although we are interested only in the determination of the equation of motion for the mean magnetization of the cluster.

As a result, the dynamic MFRG must be applied only to small clusters.

\section{Exact results from MFRG}

Besides the great qualitatively success achieved by applying the MFRG to a great variety of statistical systems (even by taking the simplest choice for the clusters) some expected rigorous quantities are obtained in some particular cases, as we have already seen in the previous section. That is what happens, for instance, when treating the one-dimensional spin- $1 / 2$ Ising model [27], the two-dimensional spin- $1 / 2$ isotropic Heisenberg model [75] and the spin-S one-dimensional BlumeCapel model [62]. While the exact critical temperature $\left(T_{c}=0\right)$ is reproduced in all models above for clusters of any size, in some of them the exact critical exponent is only obtained in the limit of infinite cluster (except for the magnetic exponent $y_{H}$ in the Ising model and the thermal exponent $\nu$ in the $d=2$ Heisenberg model, where they are exact even for the smallest clusters).

One also obtains expected quantitative values at some special points in the global phase diagram of the Ashkin-Teller model [59, 64] and the Ising model with crossing bonds [65, 66]. Exact results are also achieved by applying the method in the study of the geometrical problem of directed self-avoiding random walk in two dimensions and the Ising model on the Bethe lattice [48]. These rigorous results reproduced by the MFRG in so many different systems such as classical, quantum and geometrical statistical problems assign indeed to the method a high reliability when studying more complex models.

\section{Static Problems Applica- tions}

The previous two sections give us an idea of the performance and potentiality of the phenomenological mean field renormalization group when used to treat some particular models. In this and in the next section we will present, in a summarized way, the applications of the method (in its various forms) to several different statistical mechanical systems. It is not the purpose here to discuss the results obtained for every studied system (which amounts to over 120 papers). Rather, just an update of the treated models by employing the method will be presented. Moreover, this section is intended to be self-contained in the sense that all the references already commented in the text is referenced here again when listing the corresponding model where it has been used.

The Ising model has been extensively studied and has also been a test system for all proposed procedures involving the MFRG approach [27, 32, 49, 50, 51, 76, $77,78]$, including a combination with the decimation procedure $[79,80]$, the effects of reaction [81] and symmetry dependent fields [82], and treatments of some extended models such as: antiferromagnetic model on triangular [57] and square lattices with external field [60]; anisotropic Ising model on a triangular lattice[68]; surface effects in pure and random semi-infinite models $[83,84,85,86,87,88,89,90,91,92,93]$; the Ising system on a compressible lattice (Domb model) [94]; and the model with crossing bonds in two-dimensions $[65,66,95]$. Additional diluted versions of the Ising model have been treated by considering the effects of different kinds of random dilution including spin-glass $[55,81,96,97,98,99,100,101,102,103,104,105$, $106,107,108,109]$, dilution in the antiferromagnetic model in an external field [110] and random field systems [55, 111].

Geometrical critical phenomena and percolation have been studied by De'Bell [43, 44] and das Neves and Kamphorst Leal da Silva [112], and some exact results have been achieved [48]. The method has also been used in the study of directed percolation hypothesis for stochastic cellular automata [113], lattice gas model [114], and nematic ordered states at low temperatures [115, 116].

Other related systems include: Ashkin-Teller model [59, 64, 117]; coupled spin-1/2 Ising models [118]; ANNNI model [119]; mixed spin- $1 / 2$ spin-1 model $[67,120]$; different versions of pure and random Potts models on infinite and semi-infinite lattices [61, 121, $122,123,124,125,126,127]$; the $Z_{4}$ spin model [128]; 
Hamiltonian version of the two-dimensional $Z(q)$ symmetric spin models [129, 130]; quenched and annealed random-bond D-vector models [131]; the planar random anisotropic model [132]; q-state clock spin-glass models [133]; classical [58, 62, 134, 135, 136] and quantum random versions of the Blume-Capel model [137]; the Blume-Emmery-Griffiths model [138]; the finite temperature SU(2) lattice gauge theory at the strong coupling limit [139]; and classical XY and Heisenberg models [140].

A great variety of quantum spin systems have already been studied from the MFRG approach. Among them we have: anisotropic Heisenberg model [75, 141, 142, 143, 144]; anisotropic Heisenberg model in a transverse field [145]; pure and random spin-1/2 $[56,146,147]$ and spin $>1 / 2[148,149,150,151]$ transverse Ising models; quantum Ising model with annealed antiferromagnetic bond randomness [152]; Ising model in random transverse field [151, 153, 154, 155, 156, 157]; random mixture of Ising and Heisenberg models [158, 159]; spin-1 anisotropic Heisenberg chain [160] and spin-1 Ising model in a transverse field [161]; quantum models applied to granular superconductors [162]; and anisotropic Heisenberg model with DzyaloshinskiiMoryia interaction [163].

More recent extensions of the method include the study of the MFRG approach with the Tsallis Statistics [164] and non ambiguous location of multicritical points [136].

\section{Dynamic Problems Applica- tions}

Although fewer in number, the DMFRG has been applied in the study of dynamics and non-equilibrium properties of some interesting systems. For instance, the classical approach of Indekeu et al [69] has been extended in the treatment of the intrinsic dynamic critical properties of quantum spin models [71]. In this case, good results for the critical exponent $z$ have been obtained when compared to others from more laborious approaches.

It was also applied to non-equilibrium problems arising from the competition between microscopic mechanisms. This is the case, for instance, of the Ising model subject to two locally competing temperatures $[165,166]$. In this model, for each temperature, the corresponding transition rate satisfies the principle of detailed balance. When the two processes are considered at the same time, a continuous phase transition is observed between steady states. By using clusters of one and two spins Marques [165] was able to determine the phase diagram of the model in the temperature versus gradient of temperature plane. She also obtained values for the critical exponent $\nu$ of this non-equilibrium model in two and three dimensions, and they compare very well with the values of the corresponding equilibrium Ising model. On the other hand, if one of the temperatures becomes negative, the ferromagnetic system can display an antiferromagnetic order [166]. The heat bath at negative temperature simulates a flux of energy into the system. The MFRG was also applied to other non-equilibrium models which exhibit steady states: non-equilibrium Ising model with competing dynamics [167], critical behavior of non-equilibrium 3-state systems [168], stochastic cellular automata in one and two dimensions [113], competition between diffusion and anihilation of many particles [169], dilution in the contact process [170], critical surface behavior of models with one absorbing state [171] and one-dimensional models with multiple absorbing configurations [172].

This methodology has also been used to study the dynamic critical behavior of a semi-infinite system of spins in a simple cubic lattice with nearest-neighbor ferromagnetic interactions. As this approach has not been published in the literature and presents some similarities with the one discussed in section II.C.3 we outline below the general procedure. It is assumed that in the surface plane of the semi-infinite system the exchange coupling is given by $K_{S}$, while all the other couplings are given by $K$. For $\rho=K_{S} / K$ greater than a value $\rho_{c}$ the surface orders before the bulk while for $\rho<\rho_{c}$ the surface orders when the bulk does. As an example, in the one-site cluster of the MFRG, we take one spin in the $i-t h$ plane, and it interacts with the symmetry breaking fields: $b_{1}^{i}(t)$ of the $i-t h$ plane, and $b_{i}^{i-1}(t)$ and $b_{1}^{i+1}(t)$ of the adjacent planes. Here, and from now on, the superscript designates the particular plane away from the $i=1$ surface. As is usual, these fields are assumed to be very small, that is, we are considering the problem in the neighborhood of the surface phase transition. The equation of motion for the magnetization taking the one-site cluster, in each plane, is then given by

$$
\frac{d m_{1}^{1}(t)}{d t}=-m_{1}^{1}(t)+4 K_{S}^{\prime} b_{1}^{1}(t)+K^{\prime} b_{1}^{2}(t),
$$

for the magnetization on the surface $i=1$ and, for $i \geq 2$,

$$
\frac{d m_{1}^{i}(t)}{d t}=-m_{1}^{i}(t)+K^{\prime}\left[b_{1}^{i-1}(t)+4 b_{1}^{i}(t)+b_{1}^{i+1}(t)\right] .
$$

Eq. (99) is a generalization of Eq. (94) for the bulk magnetization with different symmetry breaking fields in different planes and Eq. (98) is a particular case of 
relation (99) for the surface magnetization with intraplane interactions $K_{S}$. Similarly, the generalized ex- pressions for the two spin cluster contained in each plane read

$$
\begin{gathered}
\frac{d m_{2}^{1}(t)}{d t}=-\left(1-\tanh K_{S}\right) m_{2}^{1}(t)+\left(1-\tanh ^{2} K_{S}\right)\left[3 K_{S} b_{2}^{1}(t)+K b_{2}^{2}(t)\right] \\
\frac{d m_{2}^{i}(t)}{d t}=-(1-\tanh K) m_{2}^{i}(t)+\left(1-\tanh ^{2} K\right)\left[K b_{2}^{i-1}+3 K b_{2}^{i}(t)+K b_{2}^{i+1}(t)\right] .
\end{gathered}
$$

In general, the magnetization for the planes $i$ taking a small block of $N$ spins inside each layer can be written as

$$
\begin{gathered}
\frac{d m_{N}^{1}(t)}{d t}=-A_{N}^{1}\left(K_{S}\right) m_{N}^{1}(t)+\sum_{j=1}^{2} B_{N}^{j}\left(K, K_{S}\right) b_{N}^{j}(t), \\
\frac{d m_{N}^{i}(t)}{d t}=-A_{N}^{i}(K) m_{N}^{i}(t)+\sum_{j=i-1}^{i+1} B_{N}^{j}(K) b_{N}^{j}(t), \quad i \geq 2,
\end{gathered}
$$

where the explicit dependence of the coefficients $A_{N}^{i}$ and $B_{N}^{i}$ on $K$ and $K_{S}$ has been given.

By considering now two clusters of $N$ and $N^{\prime}$ spins and the scaling relations given by equations (88) and (89) for the magnetizations on each plane (and introducing adequately the exponents $y_{H s}$ and $z_{S}$ for the surface magnetization and $y_{H}$ and $z$ for the bulk quantities) one finds, after taking the derivative with respect to the time $t$, a set of linear equations relating the magnetizations and the corresponding symmetry breaking fields of different layers. The set of linear equations for the magnetizations can be written as

$$
\mathcal{A}_{N} \mathcal{M}_{N}=\mathcal{A}_{N^{\prime}} \mathcal{M}_{N^{\prime}}
$$

where $\mathcal{M}_{N}$ is a column vector composed by the layer magnetizations $m_{N}^{1}, m_{N}^{2}, m_{N}^{3}, \ldots$, and $\mathcal{A}_{N}$ is a diagonal matrix whose elements are straight related to $A_{N}^{i}$. The same holds for the smaller system. A solution of (104) is obtained by equating all the terms of the diagonal of the matrices and, as all terms for $i \geq 2$ are the same, results basically in

$$
A_{N}^{1}\left(K_{S}\right)=\ell^{-z_{S}} A_{N^{\prime}}^{1}\left(K_{S}^{\prime}\right), \quad A_{N}^{i}(K)=\ell^{-z} A_{N^{\prime}}^{i}\left(K^{\prime}\right),
$$

which gives a relation among $K_{S}, K, z$ and $z_{S}$ at criticality. The set of linear equations for the remaining symmetry breaking fields can be put in the following form

$$
\mathcal{B} \mathbf{b}=0,
$$

where $\mathbf{b}$ is the column vector composed by the symmetry breaking fields $b_{N}^{1}, b_{N}^{2}, b_{N}^{3}, \ldots$, and $\mathcal{B}$ is a threediagonal matrix whose elements, using relations (105), are just functions of $K_{S}$ and $K$ at criticality. It is easy to see that the critical coupling to give an ordered surface over a paramagnetic bulk phase is found when the determinant of matrix $\mathcal{B}$ vanishes. One then obtains an equation for the surface critical temperature as a function of $\rho=K_{S} / K$, as is usual in the MFRG approach of two parameter systems. The previous bulk dynamic properties are reproduced, as expected, for $i \gg 1$.

For the simplest finite systems of Fig. 1 it is not difficult to show from Eqs. (98)-(101) and (104)-(106) that

$$
\frac{\tanh K_{S c}}{1-3 \tanh K_{S c}}=\rho,
$$

for the surface critical temperature $K_{S c}$ as a function of the ratio $\rho$ and

$$
1-\operatorname{tanhKs}=l^{-z s}, \quad 1-\tanh K=l^{-z},
$$

from which the dynamic critical exponents are obtained. The numerical results from above equations are shown in Table VI together with those obtained by taking the plaquette of four spins. We can note that the DMFRG is easily extended to study the critical dynamic phenomena on surfaces and, in particular, furnishes a value for $\rho_{c}=1.48$ comparable to that from Monte Carlo simulations $\rho_{c}=1.52$ [173], while the mean field result is $\rho_{c}=1.25$ [36, 174]. It should also be noticed that in the present approach with rather small clusters the surface magnetic exponent $y_{H S}$ is indeterminate since it cancels out in the renormalization group equations. 
Table VI. Surface dynamic critical exponent $z_{S}$ and critical surface coupling $\rho_{c}$ for the three-dimensional Ising model according to the DMFRG.

\begin{tabular}{ccc}
$N, N^{\prime}$ & $z_{S}$ & $\rho_{e}$ \\
\hline 2,1 & 1.35 & 1.35 \\
4,2 & 1.88 & 1.48 \\
\hline Monte Carlo [1] & - & 1.52 \\
\hline
\end{tabular}

[1]Ref. [173].

\section{Related Phenomenological RG}

Different phenomenological renormalization group procedures based on exact calculations in finite systems have also been proposed in the literature. In this section some of them will be discussed, namely, the effective field renormalization and finite size scaling renormalization groups. Few other methods, which are less used or applied in approaches other than the renormalization group scheme, will be referenced in the next section. Here, as in the MFRG case, we are mainly concerned in describing the methods themselves and their interrelations and similarities, and just a list of their applications in different statistical models will be presented.

\section{A. Effective Field Renormalization Group (EFRG)}

The MFRG in its several formulations computes exactly the magnetization (order parameter) for each finite cluster of spins according to the canonical distribution (19). An alternative way of obtaining this order parameter for a Hamiltonian model system $\mathcal{H}$ has been proposed by some authors [175, 176, 177] by employing an effective field theory based on the exact generalized Callen-Suzuki identity [178, 179]

$$
\left\langle O_{n}\right\rangle=\left\langle\frac{T r_{n} O_{n} \exp -\beta \mathcal{H}_{n}}{T r_{n} \exp -\beta \mathcal{H}_{n}}\right\rangle
$$

where the partial trace $T r$ is taken over the set of $n$ spins variables specified by a finite system Hamiltonian $\mathcal{H}_{n}, O_{n}$ is the corresponding order parameter (or, in general, any other function of all the $n$ spins of the cluster) and $\langle\cdots\rangle$ indicates the usual canonical thermal average taken over the ensemble defined by the complete Hamiltonian $\mathcal{H}$. The idea is simply to replace $\mathcal{H}$ by a cluster of $n=N$ spins surrounded by fixed magnetizations at values $b$. In this way the order parameter $m=O$ in Eq. (109) can be computed by employing the exponential operator technique [180] resulting, for $b<<1$, in an equation of a similar form as before for $H=0$, namely,

$$
m_{N}=f_{N}(\mathbf{K}) b_{N}
$$

Doing the same for a smaller system $\left(N^{\prime}<N\right)$ and using the mean field renormalization group assumptions given by equations (20) and (25) one obtains the EFRG recursion relation

$$
f_{N^{\prime}}\left(\mathbf{K}^{\prime}\right)=f_{N}(\mathbf{K})
$$

from which phase diagrams and estimates of critical exponents are computed.

In order to illustrate the method let us consider the spin-1/2 Ising model and $n=1$ in Eq. (109). The Callen-Suzuki identity (109) then reads

$$
\left.m_{1}=<S_{1}\right\rangle=\left\langle\tanh \left(K^{\prime} \sum_{j=1}^{c} S_{j}\right)\right\rangle
$$

where the sum is over all nearest neighbors spins of $S_{1}$. By using the exponential operator technique [180]

$$
e^{\delta D_{x}} f(x)=f(x+\delta), \quad D_{x}=\frac{\partial}{\partial x},
$$

the Eq. (112) can be written as

$$
m_{1}=\left\langle\left. e^{\delta D_{x}} \tanh (x)\right|_{x=0}\right\rangle, \quad \delta=K^{\prime} \sum_{j=1}^{c} S_{j},
$$

and, as the hyperbolic tangent does not depend on any spin configuration one has

$$
m_{1}=\left.\left\langle\prod_{j=1}^{c} e^{K^{\prime} S_{j} D_{x}}\right\rangle \tanh (x)\right|_{x=0}=\left.\left\langle\prod_{j=1}^{c}\left[\cosh \left(K^{\prime} D_{x}\right)+S_{j} \sinh \left(K^{\prime} D_{x}\right)\right]\right\rangle \tanh x\right|_{x=0},
$$


where in the last expression we have used the van der Waerden identity for the two state system

$$
e^{a S_{i}}=\cosh (a)+S_{i} \sinh (a), \quad S_{i}= \pm 1 .
$$

The above equations are still exact relations and computation of $m_{1}$ will commonly require some approximations such as decoupling the mean values of product of spins in product of spin mean values [180]. We can also see from Eq. (115) that the mean value $m_{1}$ is strongly dependent on the number of first neighbors $c$. By taking the one spin cluster $N^{\prime}=1$ of Fig. 1(a) we see that $S_{j}=b_{1}$ for all $j$. The order-parameter for the onedimensional lattice Eq. (115) assumes then the form

$$
m_{1}=\left.\left\langle\left[\cosh \left(K^{\prime} D_{x}\right)+b_{1} \sinh \left(K^{\prime} D_{x}\right)\right]^{2}\right\rangle \tanh (x)\right|_{x=0} .
$$

Since $\left.\sinh \left(K^{\prime} D_{x}\right) \cosh \left(K^{\prime} D_{x}\right) \tanh (x)\right|_{x=0}=$ $\frac{1}{2} \tanh \left(2 K^{\prime}\right),\left.\sinh ^{2}\left(K^{\prime} D_{x}\right) \tanh (x)\right|_{x=0}=0$

and $\left.\cosh ^{2}\left(K^{\prime} D_{x}\right) \tanh (x)\right|_{x=0}=0$ one has for a homogeneous system

$$
m_{1}=\tanh \left(2 K^{\prime}\right) b_{1}
$$

It is interesting to note that the usual mean field approach $m_{1}=b_{1}$ gives the exact result $T_{c}=0$ even for the one spin cluster. In fact, this approach reproduces all the expected results for the thermodynamic properties, such as magnetic susceptibility and specific heat, of the linear chain in zero external field [181] and reflects the fact that, besides appearing at most just pair correlation functions in the thermodynamic functions, the auto-correlation $\left\langle S_{i}^{2}\right\rangle=1$ has been taken into account exactly in the van der Waerden relation (116). Analogously, for the two-dimensional square lattice one gets [180]

$$
m_{1}=\frac{1}{2}[\tanh (4 K)+2 \tanh (2 K)] b_{1},
$$

which furnishes the mean field critical temperature $K_{c}=0.324$, and for the three-dimensional cubic lattice

$$
m_{1}=\frac{3}{2^{4}}\left[\tanh \left(6 K^{\prime}\right)+4 \tanh \left(4 K^{\prime}\right)+5 \tanh \left(2 K^{\prime}\right)\right] b_{1},
$$

giving $K_{c}=0.197$ [180]. As usual, from the above simple approach one always obtains classical critical exponents (as can be seen by expanding further up to the order $\left.b_{1}^{3}\right)$.

For a two spin cluster $n=2 \mathrm{Eq}$. (109) reduces to [182]

$$
m_{2}=\frac{1}{2}\left\langle S_{1}+S_{2}\right\rangle=\left\langle\prod_{j=1}^{c-1} e^{K S_{j} D_{x}} \prod_{j^{\prime}=1}^{c-1} e^{K S_{j^{\prime}} D_{y}}\right\rangle \frac{\sinh (x+y)}{\left.\cosh (x+y)+e^{-2 K} \cosh x-y\right)},
$$

where $D_{y}=\frac{\partial}{\partial y}$ and the sum in $j$ is over the first neighbors of the spin 1 , and the sum in $j^{\prime}$ is over the first neighbors of the spin 2. It is now easy, though rather lengthy, to compute the expressions for $m_{2}$ taking the cluster of Fig. 1 (b) on different lattices. We quote below just the results for the one- and two(square)-dimensional lattices which are, respectively,

$$
\begin{gathered}
m_{2}=\frac{f(2)}{g(2)+e^{-2 K}} b_{2}, \\
m_{2}=\frac{3}{2^{4}}\left(\frac{f(6)}{g(6)+e^{-2 K}}+\frac{4 f(4)}{g(4)+e^{-2 K} g(2)}+\frac{2 f(2)}{g(2)+e^{-2 K} g(4)}+\frac{3 f(2)}{g(2)+e^{-2 K}}\right) b_{2},
\end{gathered}
$$

where $f(u)=\sinh u K$ and $g(u)=\cosh u K$. A complete set of coefficients for other types of lattices taking the smallest clusters can be found in reference [177]. Eq. (122) gives also $T_{c}=0$ for the one-dimensional case and one gets $K_{c}=0.331$ and $K_{c}=0.198$ for $d=2$ and $d=3$, respectively [182].

The EFRG obtained from Eqs. (110), (111), (118) and (122) reproduce the exact one-dimensional results, including the expected thermal critical exponent $\nu$ even for the smallest systems. In general, the corresponding renormalization recursion relations for dimensions $d>1$ give better values than the MFRG, as can be seen in Table VII, where a comparison is made on square and simple cubic Ising models. In addition, the two spin block is, within this formalism, able to distinguish the two dimensional triangular lattice from the simple cubic one (both with the same coordination number $c=6$ ) regarding the critical temperature $\left(K_{c}=0.244\right.$ for the 
triangular lattice and the exact value is $K_{c}=0.275$ [35]). This distinction comes from the fact that on a triangular lattice the total number of surrounding mean field sites for $N=2$ cluster is 8 and in the simple cubic one is 10. While Eq. (121) depends on this value, in the MFRG approach expressed by Eq. (38) it only matters the number of neighbors on each site and, in some sense, the common neighbors on the triangular lattice is counted twice. The price paid, however, for such improvements in rather small systems taking the EFRG procedure clearly reflects the more elaborate calculations needed to obtaining the order parameters. As a result, contrary to the MFRG, this drastically limits the size of the employed clusters in such a way that the bigger system used in literature is $N=4$.

Besides pure and diluted Ising models [175, 176, $177,183,184,185,186]$ the EFRG has also been applied in the study of the Ashkin-Teller model [176], surface criticality in semi-infinite Ising ferromagnets [187], classical XY and Heisenberg models [140], $O(n)$ vector ferromagnetic and antiferromagnetic models [188], the transverse Ising model $[189,190]$, and quantum spin1/2 anisotropic Heisenberg models [191, 192]. The same qualitative results have been achieved in all applications.

\section{B. Finite Size Scaling Renormalization Group (FSSRG)}

The finite size scaling renormalization group (FSSRG) has been originally proposed by de Oliveira $[28,29]$ to treat Hamiltonian systems having two energy terms. The main idea of the method is to consider quantities having zero anomalous dimension, i.e., $\phi=0$ in equation (10). For the simple Ising model in an external magnetic field defined in Eq. (1) such quantities can be given by

$$
Q(K, H)=\left\langle\operatorname{sign}\left(\frac{1}{N} \sum_{i}^{N} S_{i}\right)\right\rangle
$$

and

$R(K, H)=\left\langle\operatorname{sign}\left(\frac{1}{N_{s_{1}}} \sum_{i}^{N_{s_{1}}} S_{i}\right) \operatorname{sign}\left(\frac{1}{N_{s_{2}}} \sum_{i}^{N_{s_{2}}} S_{i}\right)\right\rangle$,

where $N=L^{d}$ is the number of spins on a ddimensional lattice and $\operatorname{sign}(x)=-1,0,1$, whether $x<0, x=0, x>0$, respectively. $s_{1}$ and $s_{2}$ are two parallel surfaces with $N_{s_{1}}=N_{s_{2}}$ and separated by a distance $L / 2$ apart when taking periodic boundary conditions. For finite lattices with open boundary conditions the surfaces $s_{1}$ and $s_{2}$ can be taken as being the top and the bottom hypersurfaces of the corresponding hypercube, respectively.
Let us consider first the quantity $Q$ and see that it has zero anomalous dimension for an infinite system. While the magnetization $m=\left\langle\left(\frac{1}{N} \sum_{i}^{N} S_{i}\right)\right\rangle$ is zero for $T>T_{c}$ and behaves as

$$
m \approx \epsilon^{\beta},
$$

when $H \rightarrow 0^{+}$and $T \rightarrow T_{c}^{-}$, the quantity $Q=+1$ for $T<T_{c}$ and is zero above $T_{c}$ meaning that $Q=\epsilon^{0}$ and, from Eq. (7), $\phi=0$. Regarding now the second quantity $R$ we have that the probability of find$\operatorname{ing} \frac{1}{N_{s_{1}}} \sum_{i}^{N_{s_{1}}} S_{i}$ and $\frac{1}{N_{s_{2}}} \sum_{i}^{N_{s_{2}}} S_{i}$ with the same sign is greater than finding them with opposite signs since, according to the weak version of the Griffiths inequality [193], one has $\left\langle S_{i} S_{j}\right\rangle \geq 0$ for any pair of spins on the lattice. Thus, for the same reasoning, we also have a zero anomalous exponent for $R$.

By taking two finite systems of sizes $N, N^{\prime}$ and computing the above quantities for both clusters one then gets the renormalization recursion relations

$$
Q_{N^{\prime}}\left(K^{\prime}, H^{\prime}\right)=Q_{N}(K, H),
$$

and

$$
R_{N^{\prime}}\left(K^{\prime}, H^{\prime}\right)=R_{N}(K, H) .
$$

From equations above the complete renormalization flow in the $K-H$ plane can be exploited. It turns out that for the Ising model the FSSRG gives the quantitative correct behavior as $N>N^{\prime} \rightarrow \infty$ for all the fixed points $(K \rightarrow \infty, H=0),(K=0, H=0)$ and the Ising critical point $\left(K_{c}, H=0\right)$, while expected qualitative behavior is achieved for finite systems. For instance, taking $N=4$ and $N^{\prime}=2$, which are the smallest possible systems allowed by this approach, one readily gets

$$
\begin{aligned}
& \frac{\sinh \left(2 H^{\prime}\right)}{Z_{2}}=\frac{e^{4 K} \sinh (4 H)+4 \sinh (2 H)}{Z_{4}}, \\
& \frac{\cosh \left(2 H^{\prime}\right)-e^{-2 K^{\prime}}}{Z_{2}}=\frac{e^{4 K} \cosh (4 K)-1}{Z_{4}},
\end{aligned}
$$

where

$$
\begin{gathered}
Z_{2}=\cosh 2 H^{\prime}+e^{-2 K^{\prime}} \\
Z_{4}=2+e^{-4 K}+4 \cosh (2 H)+e^{4 K} \cosh (4 H),
\end{gathered}
$$

from which one obtains the values quoted in Table VIII for the Ising critical point. Results from analytical calculations on bigger lattices are also listed in that table. 
Table VII. Critical temperature and thermal critical exponent for the Ising model according to the EFRG. It is quoted in parenthesis the values from MFRG for comparison.

\begin{tabular}{||c|c|c||c|c|c||}
\hline $\mathrm{d}=2$ & \multicolumn{2}{|l|}{$\mathrm{d}=3$} \\
\hline$N, N^{\prime}$ & $K_{c}$ & $\nu$ & $N, N^{\prime}$ & $K_{c}$ & $\nu$ \\
\hline 2,1 & $0.358(0.347)$ & $1.39(1.67)$ & 2,1 & $0.206(0.203)$ & $1.37(1.50)$ \\
4,1 & $0.379(0.361)$ & $1.17(1.45)$ & 4,1 & $0.207(0.207)$ & $1.24(1.22)$ \\
4,2 & $0.371(0.370)$ & $1.01(1.28)$ & 4,2 & $0.208(0.207)$ & $1.14(1.27)$ \\
\hline & $0.441[1]$ & $1[1]$ & & $0.222[2]$ & $0.63[3]$ \\
\hline
\end{tabular}

[1]Refs. [34, 35].

[2]Refs. [37, 38, 39, 40].

[3]Refs. [38, 39, 40, 41].

(a)

(b)

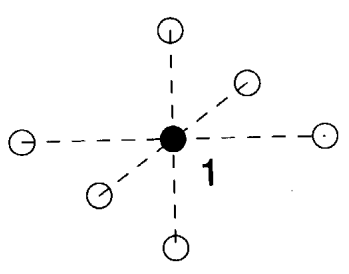

(c)

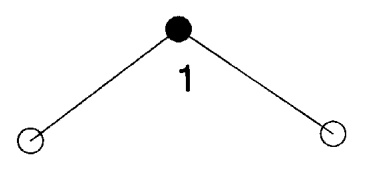

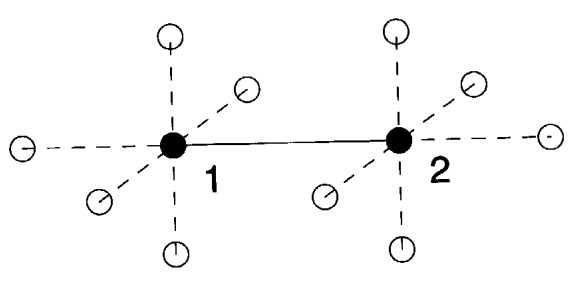

(d)

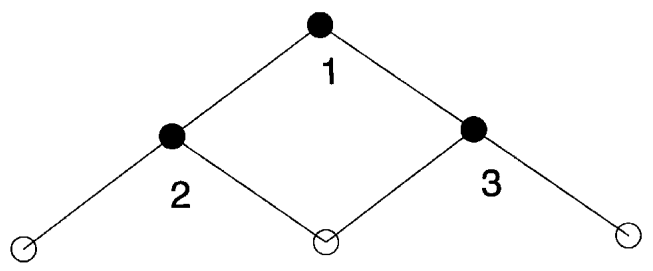

Figure 1. (a) and (b) show schematically the smallest clusters for hypercubic lattices and (c) and (d) the same for the directed self avoiding random walk (DSARW) in two dimensions. Full circles represent sites belonging to the cluster itself and open circles the corresponding surrounding sites (mean field sites).

However, contrary to the previous approaches, the FSSRG method has the great advantage of allowing Monte Carlo simulations for bigger finite systems in order to obtain the desired quantities $Q$ and $R$. Such Monte Carlo simulations are not so easily implemented and has not yet been done in the mean field like renormalization group treatments discussed herein (apart from a theory of mean field Monte Carlo simulation proposed by Netz and Berker in a different context
$[194,195,196])$. These results are also shown in Table VIII and, even taking into account the rather small computational effort, they are quite close to the exact ones. The FSSRG can also be used to treat other systems with different kinds of energy and coupling constants by conveniently choosing the quantities $Q$ and $R$ and even, in principle, be extended to study more than two parameter Hamiltonians, such as flux diagrams in three dimensions. 
Apart from the pure Ising model [28, 29, 197], the FSSRG has also been applied to the diluted Ising model [30], the spin-1 and spin-3/2 Blume-Capel model [198], and the q-state Potts model [199]. An extension to the dynamic critical behavior (for short and long times) of the Ising [200] and Potts model [201] has also been done through Monte Carlo simulations on finite systems. In this case, quite good accurate results for the dynamic critical exponents $z$ have been achieved.

It should also be mentioned that the FSSRG approach is identical to the phenomenological renormalization group proposed by Nightingale [19] when in the former one considers clusters consisting of infinite strips with finite width [202]. In order to see this equivalence let us take a strip with finite width $L$ and length $n_{L} \rightarrow \infty$ at zero external field (for simplicity). Correspondingly, the transfer matrix will be of order $2^{L} \times 2^{L}$. In this case the quantity $Q_{L}$, computed from the transfer matrix, is zero and one gets $Q_{L^{\prime}}\left(K^{\prime}, H^{\prime}=0\right)=Q_{L}(K, H=0)=0$ for any values of $L$ and $L^{\prime}<L$. On the other hand, the quantity $R_{L}$ is just a correlation function between the states of two columns separated by a distance $n_{L} / 2$ apart, which can be written as [203]

$$
R_{L}(K)=A_{L}\left(\frac{\lambda_{L}^{<}}{\lambda_{L}^{>}}\right)^{n_{L} / 2}
$$

where $A_{L}$ is a function of $K$ but independent of $n_{L}$ and $\lambda_{L}$ and $\lambda_{L}^{<}$are the greatest and second greatest eigenvalues of the corresponding transfer matrix, respectively. For two strips with $L^{\prime}<L$ one then has

$$
A_{L^{\prime}}\left(\frac{\lambda_{L^{\prime}}^{<}}{\lambda_{L^{\prime}}^{>}}\right)^{n_{L^{\prime}} / 2}=A_{L}\left(\frac{\lambda_{L}^{<}}{\lambda_{L}^{>}}\right)^{n_{L} / 2},
$$

where $n_{L}, n_{L^{\prime}} \rightarrow \infty$. Taking now the logarithm of both sides of the above equation, dividing by $n_{L^{\prime}} / 2$ and recalling that the scaling factor $\ell=L / L^{\prime}$ can also be obtained from

$$
\ell=\left(\frac{N}{N^{\prime}}\right)^{1 / d}=\left(\frac{n_{L} L^{d-1}}{n_{L^{\prime}} L^{\prime d-1}}\right)^{1 / d}=\left(\frac{L}{L^{\prime}}\right)
$$

(from which we have $\frac{n_{L}}{n_{L^{\prime}}}=\frac{L}{L^{\prime}}$ ) we finally get

$$
L^{\prime} \ln \left(\frac{\lambda_{L^{\prime}}^{<}}{\lambda_{L^{\prime}}^{>}}\right)=L \ln \left(\frac{\lambda_{L}^{<}}{\lambda_{L}^{>}}\right)
$$

which is the same as Eq. (12) since $\xi_{L}^{-1}=\ln \left(\frac{\lambda_{L}^{<}}{\lambda_{L}^{S}}\right)$ (the same for $L^{\prime}$ ).

It is interesting that two completely different phenomenological renormalization group procedures have the same (quite good) results for the properties of one parameter critical models. One can think of the FSSRG as a generalization of the original Nightingale renormalization group for completely finite systems (a fact that, in some way, explains the accuracy of the FSSRG critical values, specially in the Ising case). On the other hand, flux diagrams for two-dimensional parameter Hamiltonians (for instance, the Blume-Capel model [204]) taking infinite strips within FSSRG can be readily obtained (which is not possible from the Nightingale procedure).

Another real space renormalization group approach preserving the two spin correlation function in finite clusters proposed by Tsallis et al $[18,21]$ has also been shown to be connected to the Nightingale procedure. However, it seems rather difficult to implement the calculations by taking infinite self-dual clusters in the former method.

\section{Final Remarks}

In the above discussion we have been mainly concerned with renormalization group procedures based on finite size scaling hypothesis having a relatively wide application in rather different statistical mechanics problems. There are, however, some other renormalization group approaches based on finite lattice calculations. One of them, still close in spirit to the ones we have been described so far, is the new mean field renormalization group [205] transformation (NMFRG). To briefly illustrate this method let us recall Eq. (19) in the mean field approximation $m_{N}=b$. Close to the mean field transition $T_{c}^{N}$ the order parameter can be expanded as

$$
m_{N}=F_{N}(K) \epsilon_{N}^{\beta^{*}}+G_{N}(K) H \epsilon_{N}^{-\gamma^{*}},
$$

where $\epsilon=\left(K-K_{c}^{N}\right) / K, K_{c}^{N}$ is the mean field critical temperature and $\beta^{*}$ and $\gamma^{*}$ are the usual mean field exponents $\left(\beta^{*}=1 / 2\right.$ and $\left.\gamma^{*}=1\right)$. At $K=K_{c}^{N}$ the functions $F_{N}(K)$ and $G_{N}(K)$ are called anomaly coefficients, introduced by Suzuki $[206,207]$ and from which non-classical exponents are also achieved (the socalled coherent anomaly method - CAM). In the present renormalization group context one expands the order parameter for two different clusters $N^{\prime}<N$ and assumes a scaling relation of the form given in Eq. (20) for the approximate order parameters and the same relation for the quantities $\epsilon_{N}^{\beta^{*}}$ and $\epsilon_{N^{\prime}}^{\beta^{*}}$, i.e., $\epsilon_{N^{\prime}}^{\beta^{*}}=\ell^{d-y_{H}} \epsilon_{N}^{\beta^{*}}$. 
So, in the same way as previously done for the MFRG one then gets

$$
F_{N^{\prime}}\left(K^{\prime}\right)=F_{N}(K),
$$

from which the critical fixed point $K_{c}=K^{\prime}=K$ is obtained, as well as the thermal exponent $\nu$, whereas the corresponding magnetic exponent is given by

$$
\ell^{y_{H}}=\left(\frac{\epsilon_{N^{\prime}}}{\epsilon_{N}}\right)^{\beta^{*}+\gamma^{*}} \frac{G_{N}\left(K_{c}\right)}{G_{N^{\prime}}\left(K_{c}\right)} .
$$

It is apparent that although the anomaly coefficients are based on the classical values $\beta^{*}$ and $\gamma^{*}$, non-classical exponents are extracted from the linearization of the recursion relation (138) around the fixed point. This approach gives quite good results for the Ising model as well as for geometrical phase transitions [205], and can also be extended by using the effective field theory through the use of Callen-Suzuki identity [208]. It is also verified that the larger the value of $N$ and $N^{\prime}$, the better the approximation since more fluctuations are included in the mean field calculations within each cluster.

Just to mention, another approach, used in a different context, is the density matrix renormalization group [209, 210] which gives accurate numerical results in studying one-dimensional quantum lattice models [211], the two-dimensional Ising model [212] and strongly correlated electron systems [213]. Nevertheless, it is clear that other phenomenological renormalization group proposals can still be done by exploiting relation (10) for quantities where $\phi$ is known in the infinite system.

Concerning the present schemes there are still some open problems whose study would be natural exten- sions of the methods discussed herein. Among them, for instance, we have:

(i) there is no unified approach (as in the MFRG case) to the SBMFRG (and its corner field partner) in order to get complete flux diagrams for models with more than one Hamiltonian parameter space. In fact, just for the Ashkin-Teller model a flow diagram in the Hamiltonian parameter space has been obtained through the SBMFRG [64];

(ii) there is also a lack of a SBMFRG scheme to treat critical dynamics by considering three clusters. Only the two cluster approach has been implemented in the MFRG to dynamics;

(iii) it would be interesting to extend the EFRG procedure to account for obtaining flow diagrams;

(iv) flow diagrams in more than two Hamiltonian parameter space has not yet been considered by using neither the MFRG nor the FSSRG approach;

(v) finally, Table IX shows roughly a picture of the models treated by the MFRG approach presented in sections III-V. It is also presented the applications of the other procedures discussed herein. So, besides new systems to be treated and studied from the present approaches, all the lacunae in Table IX are straight extensions to be done on this subject. Perhaps, some readers will find it easy to readily fill in some gaps.

Table VIII. Critical temperature and thermal critical exponent for the Ising model according to the FSSRG. The figures for $N=4096$ and $N^{\prime}=1024$ are from Monte Carlo simulations with the parenthesis indicating the first uncertain digit, according to statistical fluctuations [1].

\begin{tabular}{||c|c|c||c|c|c||}
\hline \multicolumn{1}{||l|}{$\mathrm{d}=2$} & \multicolumn{1}{l|}{$\mathrm{d}=3$} & \\
\hline$N, N^{\prime}$ & $K_{c}$ & $\nu$ & $N, N^{\prime}$ & $K_{c}$ & $\nu$ \\
\hline 4,2 & 0.473 & 0.905 & 8,2 & 0.225 & 1.052 \\
16,4 & 0.432 & 1.053 & & & \\
4096,1024 & $0.440(8)$ & $1.00(0)$ & & & \\
\hline & $0.4407[2]$ & $1[2]$ & & $0.222[3]$ & $0.63[4]$ \\
\hline
\end{tabular}

[1]Data from reference [28].

[2] Refs. [34, 35].

[3]Refs. [37, 38, 39, 40].

[4]Refs. [38, 39, 40, 41]. 
Table IX. Some models studied by means of the methods described in the text (indicated by " $\mathrm{x}$ ").

\begin{tabular}{|c|c|c|c|c|}
\hline model & MFRG & EFRG & FSSRG & NMFRG \\
\hline geometrical & $\mathrm{x}$ & & & \\
\hline \multicolumn{5}{|l|}{ Classical (static) } \\
\hline pure Ising like & $\mathrm{x}$ & $\mathrm{x}$ & $\mathrm{x}$ & $\mathrm{x}$ \\
\hline random Ising like & $\mathrm{x}$ & $\mathrm{x}$ & $\mathrm{x}$ & $\mathrm{x}$ \\
\hline surface critical & $\mathrm{x}$ & $\mathrm{x}$ & & \\
\hline spin-glass & $\mathrm{x}$ & & & \\
\hline random-fields & $\mathrm{x}$ & & & \\
\hline $\mathrm{XY}$ and Heisenberg & $\mathrm{x}$ & $\mathrm{x}$ & & \\
\hline Ashkin-Teller & $\mathrm{x}$ & $\mathrm{x}$ & & \\
\hline ANNNI & $\mathrm{x}$ & & & \\
\hline mixed spin & $\mathrm{x}$ & & & \\
\hline $\mathrm{Z}(\mathrm{q})$ symmetric spin & $\mathrm{x}$ & & & \\
\hline random D-vector & $\mathrm{x}$ & & & \\
\hline$q$-state clock spin-glass & $\mathrm{x}$ & & & \\
\hline Potts & $\mathrm{x}$ & & $\mathrm{x}$ & \\
\hline Blume-Capel & $\mathrm{x}$ & & $\mathrm{x}$ & \\
\hline lattice gas & $\mathrm{x}$ & & & \\
\hline liquid crystal & $\mathrm{x}$ & & & \\
\hline Tsallis Statistics & $\mathrm{x}$ & & & \\
\hline \multicolumn{5}{|l|}{ Quantum (static) } \\
\hline pure and random transverse Ising & $\mathrm{x}$ & $\mathrm{x}$ & & \\
\hline anisotropic Heisenberg & $\mathrm{x}$ & $\mathrm{x}$ & & \\
\hline random-mixture & $\mathrm{x}$ & & & \\
\hline Dzyaloshinskii-Moryia & $\mathrm{x}$ & & & \\
\hline granular superconductors & $\mathrm{x}$ & & & \\
\hline \multicolumn{5}{|l|}{ Dynamic } \\
\hline Ising like & $\mathrm{x}$ & & $\mathrm{x}$ & \\
\hline Potts & & & $\mathrm{x}$ & \\
\hline contact-process & $\mathrm{x}$ & & & \\
\hline surface critical & $\mathrm{x}$ & & & \\
\hline quantum spin & $\mathrm{x}$ & & & \\
\hline cellular automata & $\mathrm{x}$ & & & \\
\hline
\end{tabular}

\section{Acknowledgements}

The authors would like to thank J. Ricardo de Souza, P. M. C. de Oliveira, J. Kamphorst Leal da Silva and J. G. Moreira for valuable discussions. Financial support from CNPq, FINEP, CAPES and FAPEMIG (Brazilian Agencies) is also gratefully acknowledged.

\section{References}

[1] K. G. Wilson, Renormalization group and critical phenomena. I. Renormalization group and Kadanoff scaling picture, Phys. Rev. B 4, 3174 (1971); Renormalization group and critical phenomena. II. Phase-space cell analysis at critical behavior, ibidem 3184 .

[2] K. G. Wilson, The renormalization group, Rev. Mod. Phys. 773 47, (1975); see also Problems in physics with many scales of length, Sc. Am. 241, 140 (1979).
[3] For a review, see for example, Bambi Hu, Introduction to real-space renormalization group methods in critical and chaotic problems, Phys. Rep. 91, 233 (1982).

[4] A.-L. Barabasi and H. E. Stanley, in Fractal Concepts in Surface Growth, Cambridge (1995).

[5] H. Saleur, C. G. Sammis and D. Sornette, Renormalization group of earthquakes, Nonlinear Processes Geophys. 3, 102 (1996).

[6] L. P. Kadanoff, Scaling laws from Ising models near $T_{c}$, Physics 2, 263 (1966).

[7] K. G. Wilson and M. E. Fisher, Critical exponents in 3.99 dimensions, Phys. Rev. Lett. 28, 240 (1972).

[8] E. Brézin, J. C. Le Guilou and J. Zinn-Justin, Field theoretical approach to critical phenomena, in Phase Transitions and Critical Phenomena, vol. 6 (London; Academic) ed C. Domb and M. S. Green (1976).

[9] D. J. Wallace and R. K. P. Zia, The renormalization group approach to scaling in physics, Rep. Prog. Phys. 41, 1 (1978). 
[10] Th. Niemeijer and J. M. J. van Leeuwen, Wilson theory for spin systems on triangular lattice, Phys. Rev. Lett. 31, 1411 (1973). See also Renormalization theory for Ising-like spin systems, in Phase Transition and Critical Phenomena, vol. 6 (London, Academic) ed. C. Domb and M. S. Green (1976).

[11] See, for instance, T. W. Burkhardt and J. M. J. van Leeuwen, eds., in Real Space Renormalization (Springer, Berlin, 1982).

[12] B. B. Mandelbrot, The Fractal Geometry of Nature, W. H. Freeman, San Francisco, (1982).

[13] D. Sornette, Discrete scale invariance and complex dimensions, Phys. Rep. 297, 239 (1998).

[14] J. M. Yeomans, in Statistical Mechanics of Phase Transition, (Oxford, Clarendon Press, 1992).

[15] H. J. Maris and L. P. Kadanoff, Teaching the renormalization group, Am. J. Phys. 46, 652 (1978).

[16] A. A. Migdal, Zh. Eksp. Fiz. 69, 810 and 1457 (1975); A. A. Migdal, Phase transitions in gauge and spin lattice systems, Sov. Phys. - JETP 42, 743 (1976); L. P. Kadanoff, Notes on Migdal's recursion formulas, Ann. Phys., NY 100, 359 (1976).

[17] R. H. Swendsen, Monte Carlo renormalization group, Phys. Rev. Lett. 42, 859 (1979); R. H. Swendsen, Monte Carlo renormalization group studies of the $d=2$ Ising model, Phys. Rev. B 20, 2080 (1979).

[18] C. Tsallis, A. M. Mariz, A. L. Stella, L. R. da Silva, Criticality of the discrete $N$-vector ferromagnet in planar self-dual lattices, J. Phys. A 23, 329 (1990).

[19] M.P. Nightingale, Scaling theory and finite systems, Physica 83A, 561 (1976).

[20] see R. H. Swendsen, in Real Space Renormalization T. W. Burkhardt and J. M. J. van Leeuwen, eds., (Springer, Berlin, 1982).

[21] C. Tsallis and A. C. N. de Magalhães, Pure and random Potts-like models: real space renormalization group approach, Phys. Rep. 268, 305 (1996).

[22] See, for example, J. C. Xavier, F. C. Alcaraz. D. Penã Lara and J. A. Plascak, The critical behavior of the spin-3/2 Blume-Capel model in two dimensions, Phys. Rev. B 57, 11575 (1998).

[23] B. Widom, Equation of state in the neighborhood of the critical point, J. Chem. Phys. 43, 3898 (1965).

[24] M. E. Fisher, in Proceedings of the International School Enrico Fermi, course LI, Critical Phenomena, M. S. Green, ed., Varenna, Italy (Academic Press, New York, 1971). See also M. E. Fisher and M. N. Barber, Scaling theory for finite-size effects in the critical region, Phys. Rev. Lett. 28, 1516 (1972).

[25] M. N. Barber, Finite size scaling, in Phase Transition and Critical Phenomena, vol. 8 (London, Academic) ed. C. Domb and J. L. Lebowitz (1983).

[26] R. R. dos Santos and L. Sneddon, Finite-size scaling transformations, Phys. Rev. B 23, 3541 (1981).

[27] J. O. Indekeu, A. Maritan and A. L. Stella, Renormalization group recursions by mean-field approximations, J. Phys. A 15, L291 (1982).
[28] P. M. C. de Oliveira, Finite size scaling renormalization group, Europhys. Lett. 20, 621 (1992).

[29] P. M. C. de Oliveira, RG based only on finite size scaling, Physica A 205, 101 (1994).

[30] J. M. Figueiredo Neto, S. M. Moss de Oliveira and P. M. C. de Oliveira, Finite size scaling RG: detailed description and applications to diluted Ising systems, Physica A 206, 463 (1994).

[31] M. P. Nightingale, Transfer matrix, phase transitions, and critical phenomena: numerical methods and applications, in Finite Size Scaling and Numerical Simulations of Statistical Systems, ed. V. Privman (World Scientific, Singapore, 1990).

[32] K. Croes and J. O. Indekeu, Mean-field renormalization group: Bibliography and new applications using large clusters, Mod. Phys. Lett. B (Singapore) 7, 699 (1993).

[33] H. A. Bethe, Statistical theory of superlattices, Proc. Roy. Soc. (London) A150, 552 (1935).

[34] L. Onsager, A two-dimensional model with an orderdisorder transition, Phys. Rev. 65, 117 (1944).

[35] R. J. Baxter, Exactly Solved Models in Statistical Mechanics (Academic: London 1982).

[36] K. Binder, Critical behavior at surfaces, in Phase Transitions and Critical Phenomena, eds. C. Domb and J. L. Lebowitz, vol. 8, (Academic, London, 1983).

[37] C. Domb, Ising model, in Phase Transitions and Critical Phenomena, eds. C. Domb and M. S. Green, Vol. 3, (Academic, London, 1974).

[38] D. P. Landau, Computer simulation studies of critical phenomena, Physica A 205, 41 (1994).

[39] H. W. J. Bloete, E. Luijten and J. R. Heringa, Ising universality in three dimensions: a Monte Carlo study, J. Phys. A 28, 6289 (1995).

[40] A. L. Tapalov and H. W. J. Bloete, The magnetization of the 3D Ising model, J. Phys. A 29, 5727 (1996).

[41] J. C. Le Guillou and J. Zinn-Juntin, Critical exponents from field theory, Phys. Rev. B 21, 3976 (1980).

[42] D. Stauffer and A. Aharony, in Introduction to Percolation Theory (Taylor and Francis, London) second ed. (1992).

[43] K. De'Bell, Mean field renormalization group transformation for geometric phase transitions, J. Phys. A 16, 1279 (1983).

[44] K. De'Bell and T. Lookman, Mean field renormalization group calculations for directed percolation on the square lattice, J. Phys. A 17, 2733 (1984).

[45] S. Sarbach, Phase diagram of random spin systems: II. Bethe approximation, J. Phys. C 13, 5059 (1980).

[46] J. W. Essam, Percolation theory, Rep. Prog. Phys. 43, $833(1980)$.

[47] B. K. Chakrabarti and S. S. Manna, Critical behavior of directed self-avoiding walks, J. Phys. A 16, L113 (1983). 
[48] A. das Neves, J. Kamphorst Leal da Silva and J. A. Plascak, Some exact results from the mean field renormalization group, Physica A 189, 367 (1992).

[49] J. O. Indekeu, A. Maritan and A. L. Stella, Mean field renormalization group: unified approach to bulk and surface critical behavior, Phys. Rev. B 35, 305 (1987).

[50] P. A. Slotte, Length scaling in mean field renormalization group, J. Phys. A 20, L177 (1987).

[51] J. O. Indekeu and G. Menu, Corner critical exponents from the mean-field renormalization group, J. Phys. A 21, L523 (1988).

[52] J. H. Cardy, Critical behavior at an edge, J. Phys. A 16, 3617 (1983).

[53] M. Blume, Theory of first-order magnetic phase change in $\mathrm{UO}_{2}$, Phys. Rev. 141, 517 (1966).

[54] H. W. Capel, On the possibility of first-order phase transition in Ising systems of triplet ions with zero field splitting. I. Physica 32, 966 (1966); II. ibidem 33, 295 (1967); III. ibidem 37, 423 (1967).

[55] M. Droz, A. Maritan and A. L. Stella, Mean field like renormalization group for disordered systems, Phys. Lett. A 92, 287 (1982).

[56] J. A. Plascak, Mean field renormalization group for the disordered transverse Ising model, J. Phys. A 17, L279 (1984).

[57] P. A. Slotte, Mean-field renormalization group transformation for the triangular Ising antiferromagnet, J. Phys. A 17, L85 (1984).

[58] O. F. de Alcântara Bonfim, Mean field renormalization group analysis of the Blume-Capel model, Physica A 130, 367 (1985).

[59] J. A. Plascak and F. C. Sá Barreto, Critical properties of the Ashkin-Teller model from the mean-field renormalization group approach, J. Phys. A 19, 2195 (1986).

[60] P. A. Slotte, in Phase Properties of Some Low Dimensional Models, Phd Thesis, Institutt for Teoretisk Fysikk, Norges Tekniske Høgskole, Trondheim, Norway, (1987).

[61] M. C. Marques, M. A. Santos and J. M. B. Oliveira, Renormalization group treatment of a random Potts model with different numbers of Potts states, J. Phys A 20, 5701 (1987).

[62] D. Peña Lara and J. A. Plascak, Mean field renormalization group for Ising models with $S \geq 1$, Mod. Phys. Lett. B, 101067 (1996).

[63] J. Ashkin and E. Teller, Statistics of two-dimensional lattices with four components, Phys. Rev. 64, 178 (1943).

[64] P. M. C. Oliveira and F. C. Sá Barreto, RG studies of the Ashkin-Teller model, J. Stat. Phys. 57, 53 (1989).

[65] J. A. Plascak, Renormalization group study of the twodimensional Ising model with crossing bonds, Physica A 183, 563 (1992).

[66] C. N. Likos and A. Maritan, Multi-interaction meanfield renormalization group, Phys. Rev. E 53, 3303 (1996).
[67] H. F. Verona de Resende, F. C. Sá Barreto and J. A. Plascak, Renormalization group treatment of the mixed-spin system in $d$-dimensional lattices, Physica A 149,606 (1988).

[68] C. N. Likos, Anisotropic triangular Ising model in the extended mean-field renormalization group approach, Phys. Rev. E 55, 2001 (1997).

[69] J. O. Indekeu, A. L. Stella and L. Zhang, Dynamical renormalization through classical equations of motion, J. Phys. A 17, L341 (1984).

[70] R. J. Glauber, Time dependent statistics of the Ising model, J. Math. Phys. 4, 294 (1963).

[71] J. A. Plascak, Quantum spin systems: dynamical mean field renormalization group approach, J. Phys. A 17, L697 (1984).

[72] S. K. Ma, in Modern Theory of Critical Phenomena, (New York, W. A. Benjamin, 1976).

[73] N. Jan and D. Stauffer, Determination of the non-linear relaxation exponent: a Monte Carlo study, Phys. Letts. A 93, 39 (1982).

[74] C. de Dominicis, E. Brezin and J. Zinn-Justin, Field theoretical techniques and critical dynamics: I. Ginzburg-Landau stochastic models without energy conservation, Phys. Rev. B 12, 4945 (1975).

[75] J. A. Plascak, Mean field renormalization group approach for the spin-1/2 anisotropic Heisenberg model, J. Phys. A 17, L597 (1984).

[76] J. A. Plascak and N. P. Silva, Mean field renormalization group study of the anisotropic Ising model, J. Phys. C 19, 4493 (1986).

[77] Z. P. Shi and R. B. Tao, Double-chain mean-field renormalization group approach for the Ising model, Comm. Theor. Phys. (China) 12, 333 (1989).

[78] A. Pelizzola and A. Stella, Mean field renormalization group for the boundary magnetization of strip clusters, J. Phys. A 26, 6747 (1993).

[79] O. F. de Alcântara Bonfim, F. C. Sá Barreto and M. A. Moura, Two-step renormalization group for Ising systems, J. Phys. C 17, L599 (1984).

[80] K. F. Tang and J. Z. Hu, Exact decimation transformation with mean-field approximations, J. Phys. A 19 , L621 (1986).

[81] M. Droz, A. Maritan and A. L. Stella, Mean field renormalization group with reaction field for diluted Ising model, Nuovo Cimento D 9, 123 (1987).

[82] G. Kamieniarz and R. Dekeyser, Symmetry dependent effective field, Second International School of Theoretical Physics: Symmetry and Structural Properties of Condensed Matter, 181 (1993).

[83] M. C. Marques and M. A. Santos, Mean field renormalization group study of surface effects in the Ising ferromagnet, Phys. Lett. A 118, 41 (1986).

[84] M. A. Santos, M. C. Marques and J. M. B. Oliveira, The semi-infinite Ising model with mixed ferromagnetic and antiferromagnetic interactions: a MFRG approach, J. Phys. C 21, 747 (1988). 
[85] Q. Jiang and Z. Y. Li, Critical behavior in a semiinfinite Ising ferromagnet with random field or amorphization at the surface, J. Magn. Magn. Mat. 80, 178 (1989).

[86] Q. Jiang and Z. Y. Li, Study of surface magnetism in a semi-infinite Ising ferromagnetic system, Acta Phys. Sin. (China) 38, 1062 (1989).

[87] S. L. Xu and Z. Y. Li, Surface phase transition and tricritical point of a semi-infinite Ising ferromagnet with a surface trimodal random field. J. Magn. Magn. Mat. 87, $279(1990)$.

[88] A. F. S. Moreira and W. Figueiredo, Ising model on a semi-infinite lattice: mean field renormalization group approach, Phys. Lett. A 163, 159 (1992).

[89] V. Ilkovič, Mean-field renormalization-group study of a semi-infinite Ising model with a spin-one free surface, Phys. Stat. Sol. B172, K19 (1992).

[90] Z. Y. Li, S. L. Xu, D. L. Lin and T. F. George, Surface phase diagrams of the spin-1 Ising model with a random surface field, Physica B 182, 249 (1992).

[91] Gang Sun and Jie Yang, Numerical calculations for the surface on a semi-infinite Ising model with a random surface field, Comm. Theor. Phys. 23, 25 (1995).

[92] Gang Sun, Mean-field renormalization group approaches to the surface on a semi-infinite Ising model with a random surface field, Phys. Rev. B 51, 3637 (1995).

[93] A. S. de Arruda and W. Figueiredo, Phase diagram of the semi-infinite Ising model in a random field, Mod. Phys. Lett. B 11, 973 (1997).

[94] J. A. Plascak and W. Figueiredo, Renormalization group study of compressible Ising systems, Phys. Lett. A 123, 135 (1987).

[95] J. A. Plascak, Ferromagnetic critical line of the Ising model with crossing bonds, Phys. Lett. A 154, 197 (1991).

[96] L. R. Evangelista and V. K. Saxena, Two-step renormalization group for randomly diluted Ising models, J. Phys. A 18, L389 (1985).

[97] L. R. Evangelista and V. K. Saxena, Critical properties of bond- and site-diluted triangular Ising model, Phys. Stat. Sol. B 137, K31 (1986).

[98] C. Z. Yang and Z. Y. Li, Mean field renormalization group for site-bond diluted Ising ferromagnets, Phys. Stat. Sol. B 146, 223 (1988).

[99] N. Benayad, A. Benyoussef and N. Boccara, Reentrant ferromagnetism in a two-dimensional Ising model with random nearest-neighbor interactions, J. Appl. Phys. 634001 (1988); N. Benayad, A. Benyoussef and N. Boccara, Reentrant ferromagnetism in a twodimensional Ising model with random nearest-neighbor interactions, J. Phys. C 21, 5417 (1988).

[100] A. Rosales Rivera, G. A. Perez Alcazar, and J. A. Plascak, Diluted and random-bond Ising model: application to the $F e-M n-A l$ alloys, Phys. Rev. B 41, 4774 (1990).
[101] J. A. Plascak and J. Kamphorst Leal da Silva, Renormalization group study of the two-dimensional sitediluted Ising model, Phys. Lett. A 159, 295 (1991).

[102] D. F. de Albuquerque and J. R. de Souza, Universality in the site-bond-correlated (SBC) Ising model, Phys. Lett. A 171, 421 (1992).

[103] E. Mina, A. Bohorgues, L. E. Zamora and G. A. Perez Alcazar, Diluted and random-bond Ising model for the Fe-Al disordered alloys, Phys. Rev. B 47, 7925 (1993).

[104] J. Ricardo de Souza and D. F. Albuquerque, Meanfield renormalization group: approach to diluted sitebond-correlated Ising model, Phys. Rev. B 48, 16484 (1993).

[105] A. Bohorgues, L. E. Zamora, G. A. Perez Alcazar, Theoretical phase diagram for the $\left(F e_{0.65} N i_{0.35}\right)_{1-x} M n_{x}$ system, Phys. Rev B 49, 16035 (1994).

[106] Ligia E. Zamora, G. A. Perez Alcazar, A. Bohorgues, A. Rosales Rivera and J. A. Plascak, Phase diagram of the diluted and random-bond Ising model, Phys. Rev. B 51, 9329 (1995).

[107] J. Ricardo de Souza, D. F. Albuquerque and I. P. Fittipaldi, Spin-glass phase for the diluted site-bondcorrelated version, J. Magn. Magn. Mat. 149, 269 (1995).

[108] L. E. Zamora, G. A. Perez Alcazar, A. Bohorgues, J. F. Marco and J. M. Gonzalez, Magnetic properties of the $F \epsilon_{x} M n_{0.70-x} A l_{0.30}$ alloys series, J. Appl. Phys. 82, 6165 (1997).

[109] D. F. Albuquerque and I. P Fittipaldi, Site-bondcorrelated models for the disordered magnets: application to $K N i_{p} M g_{1-p} F_{3}$, J. Appl. Phys. 81, 5297 (1997).

[110] E.E. Reinehr e W. Figueiredo, Phase diagram of the dilute antiferromagnetic Ising model, Phys.Letts. A 244, 165 (1998).

[111] A. S. de Arruda, W. Figueiredo, R. M. Sebastianes and V. K. Saxena, Field distribution and tricritical points in a random-field Ising model: Mean field renormalization group approach, Phys. Rev. B 39, 4409 (1989).

[112] A. das Neves and J. Kamphorst Leal da Silva, Twodimensional site directed percolation to bulk and surface critical properties, J. Phys. A 24, 5363 (1991).

[113] G. Odor and A. Szolnoki, Directed-percolation conjecture for cellular automata, Phys. Rev. E 53, 2231 (1996).

[114] A. Surda, A renormalization group method for lattice gas models, J. Phys. A 17, 2697 (1984).

[115] H. Leitão and M. M. Telo da Gama, A model for two dimensional orientational order. Nematic ordered states at low temperatures, Physica A 180, 263 (1992).

[116] H. Leitão and M. M. Telo da Gama, in Phase Transitions in Liquid Crystals, ed. S. Martelluci and A. N. Chester (Plenum, New York, 1992).

[117] P. Pawlicki, G. Musial, G. Kamieniarz and J. Rogiers, Mean-field renormalization group study of a modified Ashkin-Teller model, Physica A 242, 281 (1997). 
[118] L. de Cesare, I. Rawbuffo and D. I. Uzunov, Fluctuation effects in a class of systems with two order parameters, Mod. Phys. Lett. B 7, 1725 (1993).

[119] E. C. Valadares and J. A. Plascak, Mean-field renormalization group approach for the axial next-nearestneighbor Ising model, J. Phys. A 20, 4967 (1987).

[120] L. Samaj, A theory of higher order correlations: application to mixed spin systems, Physica A 161, 432 (1989)

[121] M. C. Marques and M. A. Santos, Mean field renormalization group for the Potts model in a transverse field, J. Phys. C 19, 4213 (1986).

[122] M. A. Santos and M. C. Marques, Mean field renormalization group study of the semi-infinite Potts model, J. Phys. A 20, 6143 (1987).

[123] M. C. Marques and C. A. S. Santos, Tricritical point in a random Potts model, J. Phys. A 20, 5417 (1987).

[124] M. C. Marques, M. A. Santos and J. M. B. Oliveira, Renormalization group treatment of a random Potts model with different numbers of Potts spin states, J. Phys. A 20, 5701 (1987).

[125] Q. Ye, K. F. Tang and J. Z. Hu, Application of an exact decimation transformation with mean field approximation to the Potts model, Chin. Phys. 8, 630 (1988)

[126] M. C. Marques, Three-state Potts model with antiferromagnetic interactions: A MFRG approach, J. Phys. A 21, 1061 (1988).

[127] M. C. Marques, Mean field renormalization group study of the antiferromagnetic $q$-state Potts models, J. Phys. A 21, 1297 (1988).

[128] J. A. Riera, Mean-field finite-size scaling transformation for the $Z_{4}$ spin model, J. Phys. A 17, 2885 (1984).

[129] E. Niebur and J. Sólyom, Mean field like variational and renormalization group studies of $(1+1)$ dimensional spin models, J. Phys. A 20, 2949 (1987).

[130] E. Niebur and J. Sólyom, Improved mean field like renormalization group approach for $Z(q)$ symmetric spin models, J. Phys. A 21, 539 (1988).

[131] M. L. Lyra and S. Coutinho, Phase diagrams for random-bond $D$-vector models, Physica A 155, 232 (1989).

[132] M. A. Continentino and S. Coutinho, The planar random anisotropic model: a mean-field renormalization group approach, J. Magn. Magn. Mat. 125, 49 (1993).

[133] S. T. Chen, Mean-field renormalization group for the $q$-state clock spin-glass model, Physica A 229, 181 (1996).

[134] V. Ilkovič, Mean field renormalization group study of the Blume-Capel model, Phys. Stat. Sol. B 166, K99 (1991).

[135] W. Figueiredo, Phase diagram of a spin-1 Ising model with random crystal field, Braz. J. Phys. 22, 313 (1992).

[136] D. Peña Lara and J. A. Plascak, Multicritical points from mean field renormalization group approach, Int. J. Mod. Phys. B 12, 1831 (1998).
[137] C. A. S. Santos and M. C. Marques, MFRG study of spin-1 Ising models: effects of a transverse field and randomness in the crystal-field coupling, Port. Phys. (Portugal) 18, 113 (1987).

[138] O. F. de Alcântara Bonfim and F. C. Sá Barreto, Renormalization group treatment of a spin-one Ising model with biquadratic exchange interaction, Phys. Lett. A 109, 341 (1985).

[139] Y. Günduç, A mean field renormalization group study of the finite temperature $S U(2)$ lattice gauge theory at the strong coupling limit, Phys. Lett. B 208, 115 (1988).

[140] J. Ricardo de Souza and D. F. de Albuquerque, Critical properties of the XY and classical Heisenberg models: a renormalization group study, Physica A 236, 419 (1997).

[141] D. J. Bukman and J. M. J. van Leeuwen, Mean field renormalization group for the spin-1/2 XXZ model, J. Phys. A 25, 4285 (1992).

[142] J. Ricardo de Souza, New renormalization group approach for the spin-1/2 anisotropic Heisenberg model, Phys. Lett. A 216, 321 (1996).

[143] J. Ricardo de Souza and J. A. Plascak, Phase transition in the three-dimensional anisotropic Heisenberg antiferromagnetic model, Phys. Lett. A 237, 66 (1997).

[144] J. Ricardo de Souza, Universality in the critical exponents $\delta$ and $\eta$, Phys. Lett. A 252, 137 (1999).

[145] Hai-Xia Cao, Qing Jiang, Chen Xu and Zhen-Ya Li, Critical behavior of the spin-1/2 anisotropic Heisenberg ferromagnet with a transverse field, Sol. Stat. Comm. 103, 223 (1997).

[146] E. C. Valadares and J. A. Plascak, Diluted transverse Ising model within the mean field renormalization group approach, Phys. Lett. A 114, 95 (1986).

[147] C. Z. Yang and W. J. Song, Mean field renormalization group and path-integral methods for the disordered transverse Ising model, Phys. Stat. Sol. B 162, K113 (1990).

[148] W. J. Song and C. Z. Yang, MFRG and DPIR for a $\mathrm{S}=3 / 2$ transverse Ising model in a random field, Phys. Stat. Sol. 183, 259 (1994).

[149] W. J. Song and C. Z. Yang, Study of the critical behavior for the $S=5 / 2$ transverse Ising model in a random field, Phys. Stat. Sol. 185, 227 (1994).

[150] W. J. Song and C. Z. Yang, The critical properties of the spin-3/2 Ising model with a transverse field, Sol. Stat. Comm. 91, 145 (1994).

[151] W. J. Song and C. Z. Yang, MFRG-DPIR study of spin-5/2 Ising model with a random transverse field, Sol. Stat. Comm. 92, 361 (1994).

[152] Z. P. Shi and R. B. Tao, Magnetic phase transitions in quantum Ising model with annealed antiferromagnetic bond randomness, Comm. Theor. Phys. (China) 13, 433 (1990).

[153] T. F. Cassol, W. Figueiredo and J. A. Plascak, Ising model in a random transverse field, Phys. Lett. A 160, 518 (1991). 
[154] W. J. Song, MFRG with DPIR for the disordered Ising model in a random transverse field, Sol. Stat. Comm. 89, 379 (1994).

[155] W. J. Song and C. Z. Yang, MFRG-DPIR study of the Ising model. Spin- $3 / 2$ with random and spin- $5 / 2$ with fixed transverse field, Phys. Stat. Sol. 186, 511 (1994).

[156] L. A. Serkov and V. O. Shvalev, Ising model in a random transverse field, Phys. Sol. Stat. 36, 1847 (1994).

[157] W. J. Song, MFRG-DPIR study on the arbitrary spin Ising model with random transverse field, Phys. Stat. Sol. 196, 243 (1996).

[158] M. Droz and A. Pekalski, Mean field like renormalization group study of random two-component magnetic systems, Phys. Lett. A 107, 89 (1985).

[159] M. L. Martins, J. A. Plascak and F. C. Sá Barreto, Mean field renormalization group for the spin-1/2 random anisotropic Heisenberg model, Phys. Lett. A 126, 353 (1988).

[160] J. Sólyom, Variational approach to the spin-1 anisotropic Heisenberg chain, Phys. Rev. B 32, 7524 (1985).

[161] C. Z. Yang and W. J. Song, Mean field renormalization group and path-integral methods for spin-one disordered Ising model in a transverse field, Phys. Stat. Sol. B 166, 269 (1991).

[162] E. Granato and M. A. Continentino, Charging effects and quantum crossover in granular superconductors, Phys. Rev. B 48, 15977 (1993).

[163] L. A. Serkov and V. O. Shvalev, Heisenberg ferromagnet with random Dzyaloshinskii interaction: mean field renormalization group method, Phys. Sol. Stat. 36, 98 (1994).

[164] J. Ricardo de Souza, Study of the mean field renormalization group method with the Tsallis statistics, Physica A 235, 534 (1997).

[165] M. C. Marques, Critical behavior of the nonequilibrium Ising model with locally competing temperatures, J. Phys. A 22, 4493 (1989).

[166] T. Tomé, M. J. de Oliveira and M. A. Santos, Nonequilibrium Ising model with competing Glauber dynamics: a MFRG approach, J. Phys. A 24, 3677 (1991).

[167] M. C. Marques, Non-equilibrium Ising model with competing dynamics: a MFRG approach, Phys. Lett. A 145, 379 (1990).

[168] M. C. Marques, Critical behavior of non-equilibrium three-state systems, J. of Phys. A 26, 1559 (1993).

[169] M. C. Marques, Application of the mean field renormalization group approach to non-equilibrium critical phenomena, Physica A 163, 915 (1990).

[170] M. C. Marques, Effects of dilution on the phase diagram of some non-equilibrium systems, J. Phys. A $\mathbf{2 3}$ ,3389 (1990).

[171] M. Droz, L. Frachebourg and M. C. Marques, Critical phenomena at surfaces in a model of non-equilibrium phase transitions, J. Phys. A 24, 2869 (1991).
[172] J. F. F. Mendes, R. Dickman, M. Henkel and M. C. Marques, Generalized scaling for models with multiple absorbing states, J. Phys. A 27, 3019 (1994).

[173] D. P. Landau and K. Binder, Critical behavior of the surface layer magnetism at the extraordinary transition in the three-dimensional Ising model, Phys. Rev. B 41, $4786(1990)$.

[174] K. Binder and P. C. Hohenberg, Surface effects on magnetic phase transitions, Phys. Rev. B 9, 2194 (1974).

[175] Z. Y. Li and C. Z. Yang, Effective field renormalization group study for the diluted Ising model, Phys. Rev. B 37, 5744 (1988).

[176] N. Benayad, A. Benyoussef, N. Boccara and A. E. Kenz, Renormalization group recursion relations using the application of generalized Callen indentities to the Ashkin-Teller model, J. Phys. C 21, 5747 (1988).

[177] I. P. Fittipaldi, Effective field renormalization group approach for Ising lattice spin systems, J. Magn. Magn. Mat. 131, 43 (1994).

[178] H. B. Callen, A note on Green functions and the Ising model, Phys. Lett. 4, 161 (1963).

[179] M. Suzuki, Generalized exact formulation for the correlations of the Ising model and other classical systems, Phys. Lett. 19, 267 (1965).

[180] R. Honmura and T. Kaneyoshi, Contributions to a new type of effective field theory of the Ising model, J. Phys. C 12, 3979 (1979).

[181] F. C. Sá Barreto and I. P. Fittipaldi, A simple derivation of the thermodynamic properties of the onedimensional Ising model, Rev. Bras. Fis. (Brazil) 11, 745 (1981).

[182] A. Bobak and M. Jascur, A new type of effective field theory for Ising models with spin-1/2, Phys. Stat. Sol. (b) 135, K9 (1986).

[183] V. Ilkovic, Mean field renormalization group study for the Ising model, Phys. Stat. Sol. B 166, K31 (1991).

[184] L. A. Serkov, Fixed scale transformation with allowance for short-range spin correlations, Theor. Math. Phys. 92, 759 (1992).

[185] D. F. de Albuquerque and I. P. Fittipaldi, A unified effective field renormalization group framework approach for the quenched diluted Ising models, J. Appl. Phys. 75, 5832 (1994).

[186] D. F. de Albuquerque and I. P. Fittipaldi, Effectivefield renormalization group (ERFG): phase diagrams of quenched bond-mixed spin Ising model on square and Kagome lattices, J. Mag. Mag. Mat. 104-107, 236 (1992).

[187] V. Ilkovic, Effective-field renormalization-group approach study of surface effects in the Ising Ferromagnet, Phys. Stat. Sol. B 166, K35 (1991).

[188] J. Ricardo de Souza, Equivalence of the $O(n)$ vector ferromagnetic and antiferromagnetic models, Physica A 256, 383 (1998). 
[189] Q. Jiang and Z. Y. Li, Effective field renormalization group study for the transverse Ising model in a quantum system, Phys. Rev. B 40, 11264 (1989).

[190] Jiang Xuefan and Zhong Jialin, The first fluctuation correction for the transverse Ising model, Phys. Stat. Sol. B 193, K5 (1996).

[191] J. Ricardo de Souza, Critical behavior of the quantum spin-1/2 anisotropic Heisenberg model, Physica A 259, 138 (1998).

[192] Ijaní G. Araújo, J. Cabral Neto and J. Ricardo de Souza, Effective field renormalization group approach for the spin-1/2 anisotropic Heisenberg antiferromagnetic model, Physica A, in press (1999).

[193] See D. Ruelle, in Statistical Mechanics, (Reading, W. A. Benjamin, 1976).

[194] R.R. Netz and A. N. Berker, Monte Carlo mean field theory and frustrated systems in two and threedimensions, Phys. Rev. Lett. 66, 2901 (1991); Netz and Berker reply, ibidem 67, 1808 (1991).

[195] R.R. Netz and A. N. Berker, Hard-spin mean field theory: formulation for Ising, XY and other models, J. of Appl. Phys. 70, 6074 (1991).

[196] E. F. Henriques and S. R. Salinas, Monte Carlo mean field method for spin systems, Phys. Rev. B 51, 8621 (1995).

[197] P. M. C. de Oliveira, Broad histogram method and zero dimensional quantities, to appear in Comp. Phys. Comm. (1999).

[198] S. Moss de Oliveira, P. M. C. de Oliveira and F. C. Sá Barreto, The spin S Blume-Capel model flow diagram, J. Stat. Phys. 78, 1619 (1995).

[199] P. M. C. de Oliveira, S. Moss de Oliveira, C. E. Cordeiro and D. Stauffer, Finite size scaling for firstorder transitions: Potts model, J. Stat. Phys. 80, 1433 (1995).

[200] M. Silvério Soares, J. Kamphorst Leal da Silva and F. C. Sá Barreto, Numerical methods to evaluate the dynamical critical exponent, Phys. Rev. B 55, 1021 (1997).

[201] M. Silvério Soares, in Study of the spin critical dynamics through finite size scaling, $\mathrm{PhD}$ thesis (in portuguese) Universidade Federal de Minas Gerais, Departamento de Física, unpublished (1997).
[202] J. A. Plascak and J. Kamphorst Leal da Silva, On the equivalence of finite size scaling renormalization group and phenomenological renormalization, Physica A 233, 551 (1996).

[203] C. Domb, Cooperative phenomena in crystals, Ad. Phys. 9, 149 (1969).

[204] L. de Matos Castro and J. A. Plascak, The BlumeCapel model from finite size scaling $R G$ with infinite strips, to be published.

[205] J. Kamphorst Leal da Silva, R. Alves Nogueira, J. A. Plascak and M. Droz, A new mean field renormalization group transformation, J. Phys. A 27, 1495 (1994).

[206] M. Suzuki, Statistical mechanical theory of cooperative phenomena. I. General theory of fluctuations, coherent anomalies and scaling exponents with simple applications to critical phenomena, J. Phys. Soc. Japan 55, 4205 (1986).

[207] M. Suzuki, M. Katori and X. Hu, Coherent anomaly method in critical phenomena. I., J. Phys. Soc. Japan 56, 3092 (1987).

[208] F. O. Coelho and J. A. Plascak, The new mean field renormalization group from effective field theory, Mod. Phys. Lett. B 9, 1617 (1995).

[209] S. R. White, Density matrix formulation for quantum renormalization groups, Phys. Rev. Lett. 69, 2863 (1992).

[210] S. R. White, Density matrix algorithms for quantum renormalization groups, Phys. Rev. B 48, 10345 (1993).

[211] R. M. Noack, S. R. White and D. J. Scalapino, Correlations in a two-chain Hubbard model, Phys. Rev. Lett. 73, 882 (1994).

[212] Tomotoshi Nishino, Density matrix renormalization group method for $2 D$ classical models, J. Phys. Soc. Jp. 64, 3598 (1995).

[213] S. R. White, Strongly correlated electrons systems and the density matrix renormalization group, Phys. Rep. 301, 187 (1998). 